\title{
Stochastic determination of entrainment risk in uniformly sized sediment beds at low transport stages:
}

\section{Experiments}

\author{
Matteo Tregnaghi, ${ }^{1}$ Andrea Bottacin-Busolin, ${ }^{2}$ Simon Tait, ${ }^{1}$ and Andrea Marion ${ }^{3}$ \\ Received 28 June 2011; revised 22 August 2012; accepted 24 August 2012; published 5 October 2012.
}

[1] Fluvial sediment transport is caused by a complex interaction of interdependent grain and fluid processes many of which are stochastic in nature and cannot be adequately represented by deterministic equations. Random variable analysis has been used previously but limited data are available to describe the variability of grain resistance combined with particle arrangements, and thus validate such analysis. In this study low to medium bed load transport tests were carried out in a flume where sediment movement was monitored using a three-camera 3D PIV system. Simultaneous grain motion and flow velocity measurements were made on a plane located slightly above and parallel to the sediment bed. Detailed statistical velocity information was acquired to model the velocity distribution at the bed level. This was combined with the joint probabilistic distribution of particle exposures and grain resistance to motion, which were obtained from discrete particle modeling (DPM) simulations. DPM simulations were used to provide a stochastic mathematical description of the risk that a stationary particle is entrained by the flow. Predictions from the stochastic model equations replicated the observed pulsation in sediment transport. This demonstrates that it is possible to simulate sediment entrainment and transport at a high resolution by adequately modeling all the sub-processes. A number of flow patterns were identified that caused large fluctuations of the entrainment rate. These all exhibit high velocity flow structures, but they selectively cause the dislodgement of individual particles located at different positions. This selective behavior follows from the variability of the interaction between the near-bed flow and the particles having different exposure.

Citation: Tregnaghi, M., A. Bottacin-Busolin, S. Tait, and A. Marion (2012), Stochastic determination of entrainment risk in uniformly sized sediment beds at low transport stages: 2. Experiments, J. Geophys. Res., 117, F04005, doi:10.1029/2011JF002135.

\section{Introduction}

[2] Both experimental and field observations [Drake et al., 1988; Nelson et al., 1995; Papanicolaou et al., 2001; Ancey et al., 2006; Radice et al., 2009; Lajeunesse et al., 2010] and studies based on numerical simulations of particle trajectories [Schmeeckle and Nelson, 2003; Wu and Jiang, 2007] have shown that the character of sediment motion at low transport stages is highly intermittent. Grains move for a period of time followed by a rest phase both apparently characterized by randomly distributed durations. One significant aspect is that

\footnotetext{
${ }^{1}$ School of Engineering, Design \& Technology, University of Bradford, Bradford, UK.

${ }^{2}$ Department of Land and Water Resources Engineering, KTH Royal Institute of Technology, Stockholm, Sweden. Italy.

${ }^{3}$ Department of Industrial Engineering, University of Padua, Padua,

Corresponding author: M. Tregnaghi, School of Engineering, Design \& Technology, University of Bradford, Chesham Building, Richmond Road, Bradford BD7 1DP, UK. (tregnaghi@wetengineering.com)

C2012. American Geophysical Union. All Rights Reserved. 0148-0227/12/2011JF002135
}

the fluid is unable to dislodge particles for most of the time and that the frequency of the turbulent flow events appears to control the frequency or the likelihood of the associated entrainment event [Cao, 1997; Dancey et al., 2002]. Field and laboratory experiments by Church et al. [1998] and Marion et al. [2003] indicated that significant stability could be related to the grain scale surface geometry of water-worked beds. These observations, combined with findings by Papanicolaou et al. [2001] who showed that the presence of various roughness geometries in gravel bed streams affects the turbulence characteristics of the flow, suggest that the self-organization in a water-worked sediment bed surface has an impact on both grain stability and the near bed turbulence structure.

[3] These observations have encouraged researchers to develop models that predict the sediment flux rate based on a probabilistic view of the entrainment process. Following the experimental work of Shvidchenko and Pender [2000] and Papanicolaou et al. [2002], the probability of entrainment may be defined as the fraction of available particles that is dislodged per turbulent event. It has been hypothesized that such a probability of entrainment can be compared with the 
(a)

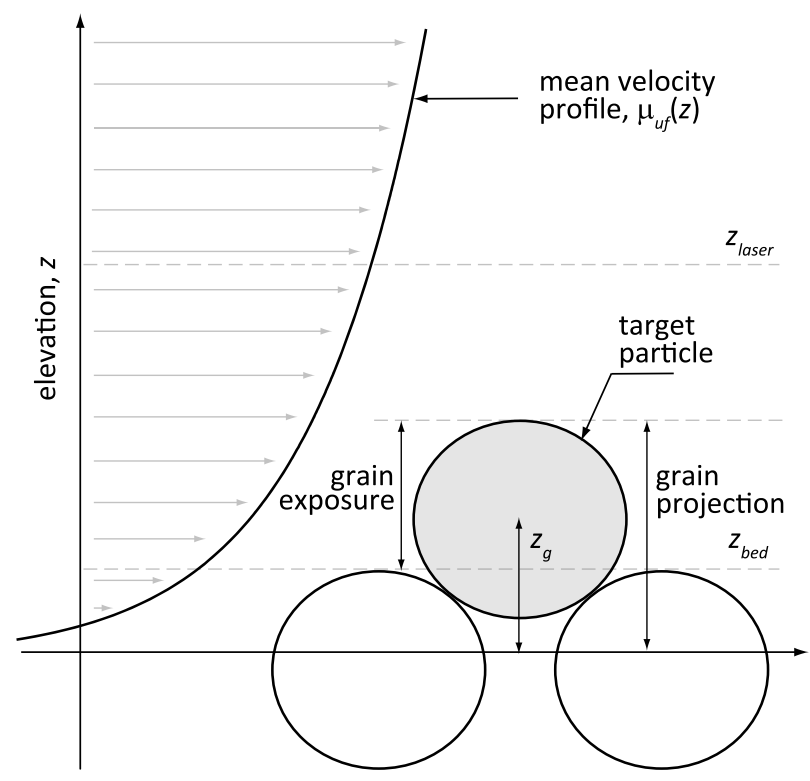

(b)

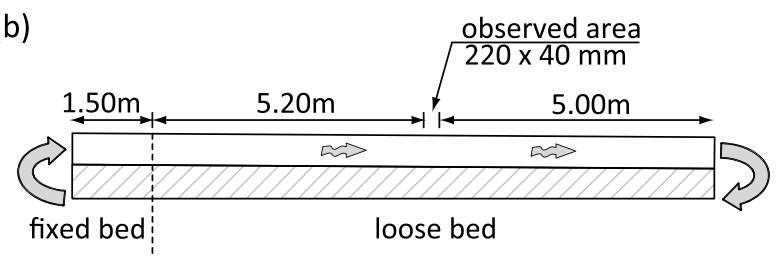

(c)

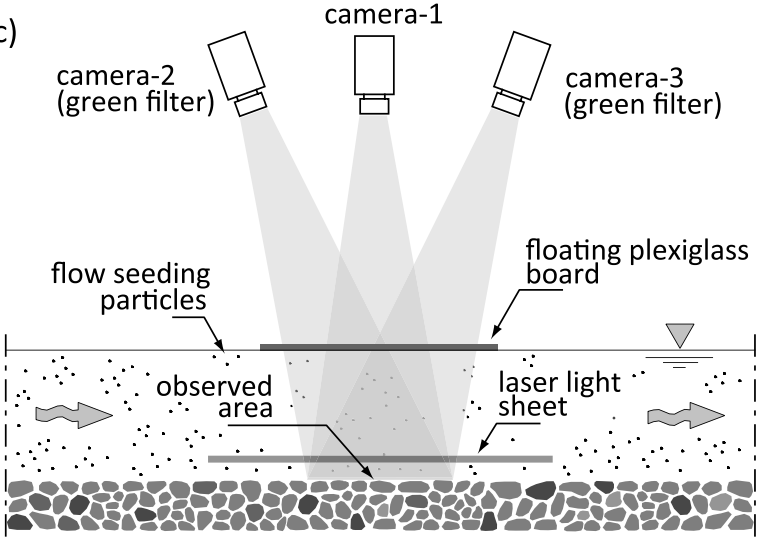

Figure 1. Experimental setup. (a) Geometrical sketch of the sediment bed and position of the measurement plane. (b) Longitudinal section of the flume. A fixed bed of gravel covered the upstream end of the flume for a distance of $1.50 \mathrm{~m}$. The remaining part of the flume was filled with a bed of loose natural river gravel with an average diameter $d_{50}=5.0 \mathrm{~mm}$. (c) Modified 3D PIV measurement system. An area of $220 \mathrm{~mm}$ by $40 \mathrm{~mm}$ located on the centerline of the flume was viewed by three cameras placed vertically above the water surface. Cameras 2 and 3 were used to obtain flow field information while Camera 1 was used to gather data on grain transport.

likelihood of the fluctuating fluid forces exceeding the resisting force or impulse [e.g., Diplas et al., 2008; Valyrakis et al., 2010]. This concept reflects the interrelationship of near-bed turbulent flow structures and bed surface properties. Schmeeckle and Nelson [2003] suggested that a key source of the highly variable nature of particle entrainment at low transport stages is the randomness of the resisting forces to downstream motion resulting from the geometrical relation of a bed grain to the grains that it rests upon. Several experimental studies [e.g., Fenton and Abbott, 1977; Kirchner et al., 1990; Buffington et al., 1992; Johnston et al., 1998], theoretical analyses [Wiberg and Smith, 1987; Dey, 1999; Cheng et al., 2003], and numerical experiments based on discrete particle models (DPM) [McEwan and Heald, 2001; Schmeeckle and Nelson, 2003; Heald et al., 2004] have all indicated that for natural sediments the local configuration of neighboring grains results in highly variable grain stability.

[4] Experimental observations that link the entrainment of individual grains with simultaneous measurements of the local flow field can significantly improve the understanding of the role of turbulence on sediment entrainment [e.g., Nelson et al., 1995; Bottacin-Busolin et al., 2008; Valyrakis et al., 2011]. Difficulties in describing surface texturing at the grain scale and in developing measurement methods that can capture spatial and temporal variability of bed material transport increment the complexity of adequately modeling these processes [e.g., Rennie and Villard, 2004]. In this study, an image based system was used to quantify the interaction between grain-scale turbulence events and sediment entrainment. Experiments were carried out using a three-camera PIV to measure simultaneous grain motion and flow velocities on a plane located slightly above and parallel to the sediment bed (Section 2). The statistics of the three velocity components were examined at the grain scale so as to obtain an accurate probabilistic description of the near-bed flow field that correlates with the entrainment of particles (Section 3). The focus of this analysis is on the stochastic aspect of sediment entrainment, which can be described in terms of 'probability' or 'risk' of entrainment $R_{E}$ as [Tregnaghi et al., 2012]

$$
\begin{aligned}
R_{E} & =P\left(\mathrm{~T}_{g}<\mathrm{T}_{f}\right) \\
& =\int_{-\infty}^{\infty} d \tau_{f} \int_{-\infty}^{\infty} f_{\mathrm{T} f}\left(\tau_{f} \mid Z_{g}=z_{g}\right) \cdot\left[\frac{\partial}{\partial z_{g}} F_{\mathrm{T} g, Z_{g}}\left(\tau_{f}, z_{g}\right)\right] d z_{g}
\end{aligned}
$$

where the random variables $\mathrm{T}_{f}, \mathrm{~T}_{g}$ and $Z_{g}$ represent the fluid shear stress, the critical shear stress, and the bed elevation of the 'target' grain, respectively. Lower-case letters indicate single occurrences of these variables. The statistical functions $F_{\mathrm{T} g, Z g}$ and $f_{\mathrm{T} f}(\cdot \cdot)$ are the cumulative joint distribution of $\mathrm{T}_{g}$ and $Z_{g}$ and the conditional probability density of $\mathrm{T}_{f}$ given the occurrence of $Z_{g}=z_{g}$, respectively. The bed elevation $Z_{g}$ is described by a probability distribution $F_{Z g}$ having a density function $f_{Z g}$. The geometrical schematic of a particle protruding into the flow is shown in Figure 1a. The risk of entrainment is estimated with the distribution of experimental fluid shear stress obtained from near-bed velocity data (Section 4). This was combined with DPM-based numerical simulations that linked the distribution of forces required to entrain grains with the statistical properties of the bed surface (Section 5). The probability of entrainment is then compared to entrainment 
Table 1. Experimental Parameters

\begin{tabular}{lccccccc}
\hline Test & $\mathrm{S}(\%)$ & $\mathrm{Q}(\mathrm{l} / \mathrm{s})$ & $\mathrm{U}(\mathrm{m} / \mathrm{s})$ & $\mathrm{R}_{\mathrm{e}}\left(\cdot 10^{5}\right)$ & $\mathrm{u}_{\mathrm{s}}(\mathrm{m} / \mathrm{s})$ & $\tau_{0}^{*}$ & $\mathrm{Q}_{\mathrm{s}}(\mathrm{g} / \mathrm{s})$ \\
\hline $\mathrm{T} 1$ & 0.55 & 40.5 & 0.88 & 2.81 & 0.067 & 0.057 & - \\
$\mathrm{T} 2$ & 0.57 & 42.6 & 0.93 & 2.93 & 0.069 & 0.059 & - \\
$\mathrm{T} 3$ & 0.59 & 43.3 & 0.94 & 2.98 & 0.070 & 0.061 & - \\
T4 & 0.62 & 43.8 & 0.95 & 3.02 & 0.071 & 0.064 & - \\
T5 & 0.65 & 44.8 & 0.97 & 3.10 & 0.074 & 0.068 & 3.1 \\
T6 & 0.69 & 45.6 & 0.99 & 3.16 & 0.076 & 0.072 & - \\
T7 & 0.72 & 46.4 & 1.01 & 3.22 & 0.077 & 0.074 & - \\
T8 & 0.75 & 47.2 & 1.03 & 3.28 & 0.079 & 0.077 & - \\
T9 & 0.77 & 48.1 & 1.05 & 3.34 & 0.080 & 0.080 & 3.4 \\
T10 & 0.80 & 49.0 & 1.07 & 3.40 & 0.081 & 0.083 & - \\
T11 & 0.83 & 49.8 & 1.08 & 3.45 & 0.083 & 0.086 & 4.0 \\
T12 & 0.86 & 50.9 & 1.11 & 3.53 & 0.085 & 0.090 & - \\
\hline
\end{tabular}

rate measurements collected with the flow field synchronized sediment-tracking system (Section 6). This comparison aims to examine the influence of the constituent sub-processes leading to equation (1) on the random nature of sediment entrainment.

\section{Experiments}

[5] A $12 \mathrm{~m}$ long, $0.5 \mathrm{~m}$ wide tilting flume was equipped with a measurement system that enabled simultaneous high resolution measurement of sediment entrainment and velocities within a plane close to the deposit surface. A fixed bed of gravel covered the upstream end of the flume for a distance of $1.50 \mathrm{~m}$. This ensured the development of a stable upstream boundary layer for all the experiments. The remaining part of the flume was filled with a bed of loose natural river gravel with an average diameter $d_{50}=5.0 \mathrm{~mm}$, standard deviation $\sigma_{g}=1.3$, and density $\rho_{s}=2650 \mathrm{~kg} / \mathrm{m}^{3}$ (see Figure $1 \mathrm{~b}$ ). The sediment bed was prepared to produce a well-mixed deposit of uniform thickness with no bed forms. Afterwards, the flume was tilted to the desired slope, a downstream weir level was set and a constant water discharge was imposed to obtain steady and uniform hydraulic conditions. Conditions were selected so that individual grain entrainments and depositions could be clearly identified and measured velocity perturbations could be unambiguously linked to individual grain events.

[6] An area of $220 \mathrm{~mm}$ by $40 \mathrm{~mm}$ located on the centerline of the flume, $6.70 \mathrm{~m}$ from the inlet, was used for measurement. This area was viewed by three cameras placed vertically above the water surface as illustrated in Figure 1c. A floating glass sheet was placed at the same level as the design water depth to avoid image distortion due to water surface oscillations. The measurement area size was selected to provide velocity data at a suitable spatial scale (i.e., one interrogation area to correspond to at least one grain plan area), and to allow observation of enough grain movements during each experiment so that statistically significant data on grain entrainment could be obtained. Two Nd:Yag pulsed lasers combined with beamexpanding optics produced a light sheet of approximately uniform intensity. The laser sheet was located approximately at $z_{\text {laser }}=10 \mathrm{~mm}$ above and parallel to the original sediment bed to avoid interference between the flow field measurement and the moving bed load and to reduce reflected light from the bed in the flow images.

[7] Two cameras (2 and 3) had a green filter and the other camera operated using a white strobe light. Cameras 2 and 3 were used to obtain flow field information while the other camera was used to gather data on grain entrainments, depositions and movements. Cameras 2 and 3 collected pairs of images with the time step between the images $\Delta t_{p}=1 \mathrm{~ms}$ and the frequency of the collection of image pairs $f r=45 \mathrm{~Hz}$. In order to measure the spatial flow velocity pattern, spherical seeding particles were fed into the flow. These were made of polystyrene with an average diameter of $d_{p}=200 \mu \mathrm{m}$ and density $\rho_{p}=995 \mathrm{~kg} / \mathrm{m}^{3}$. Their low submerged density and inertia meant that they closely followed the flow. The multicamera stereoscopic system allowed for 3D velocity measurements on the laser illuminated plane. Camera-1 was focused on the sediment bed surface and captured an image of the bed at the starting time of the second of the paired flow images. The interrogation areas were set at $4.3 \mathrm{~mm}$ by $4.3 \mathrm{~mm}$ with an overlap of $75 \%$, leading to a square mesh grid comprised of $N=35 \times 185=6475$ nodes. This provided near bed flow velocity data at the grain spatial scale. Findings reported by Bottacin-Busolin et al. [2008] support the assumption that the observed instantaneous velocities at the measurement elevation are reasonably indicative of instantaneous entrainment velocities.

[8] Twelve tests carried out at different slopes but with the same water depth, $h_{u}=100 \mathrm{~mm}$. Flow velocity and grain motion measurements were taken simultaneously for periods of approximately $10 \mathrm{~min}$ duration during each test. Table 1 shows the main hydraulic parameters of the experimental tests: $S=$ slope of the flume, which was approximately equal to the bed slope during the recording session; $Q=$ water discharge; $U=$ depth averaged flow velocity; $R_{e}=4 R_{H} U / \nu=$ flow Reynolds number, where $R_{H}=$ hydraulic radius; $u_{s}=$ $\left(g R_{H} S\right)^{0.5}=$ shear velocity, with $g=$ acceleration due to gravity; and $\tau_{0}^{*}=\tau_{0} / g\left(\rho_{s}-\rho\right) d_{50}=$ Shields parameter, where $\tau_{0}=$ boundary shear stress and $\rho_{s}$ and $\rho=$ density of sediment and water respectively; $Q_{s}=$ sediment discharge. The experiments were run with increasing levels of boundary shear stress, so that the distribution of velocities and linked grain motions could be determined as the system moved away from the threshold of motion $\left(\tau_{0}^{*} \approx 0.057\right)$ to conditions of moderate bed load transport $\left(\tau_{0}^{*} \approx 0.090\right)$. Sediment transport and entrainment rate measurements were collected through visual inspection of the time series of bed images for tests T5, T9 and T11. At the scale of the measurement area the level of sediment motion ranged from a few to ten grains entrained per unit time, and frequent and irregular sediment pulsations around a mean value were observed during each experiment. In the next sections a detailed analysis is performed on the statistical properties of the near-bed flow field over the full range of hydraulic conditions investigated in this study, with particular reference to those tests where sediment transport was measured.

\section{Statistical Properties of the Near-Bed Flow Field}

\subsection{Probability Distributions of the Three Velocity Components}

[9] Probability density functions were obtained for the three orthogonal velocity components. The analysis of the velocity variability can provide a statistical description of the local fluid shear stress $\tau_{f}$, which is regarded as a random variable depending on both turbulent fluctuations and local 
Table 2. Summary of Statistical Parameters for Near-Bed Streamwise Velocity

\begin{tabular}{lcccc}
\hline TEST & $\mu_{\mathrm{u}, \text { laser }}(\mathrm{m} / \mathrm{s})$ & $\sigma_{\mathrm{u}, \text { laser }}(\mathrm{m} / \mathrm{s})$ & $\mathrm{S}_{\mathrm{u}, \text { laser }}(-)$ & $\mathrm{K}_{\mathrm{u}, \text { laser }}(-)$ \\
\hline T1 & $0.63 \pm 0.00$ & $0.110 \pm 0.005$ & $-0.05 \pm 0.07$ & $-0.39 \pm 0.07$ \\
T2 & $0.65 \pm 0.01$ & $0.112 \pm 0.004$ & $0.00 \pm 0.06$ & $-0.30 \pm 0.18$ \\
T3 & $0.67 \pm 0.02$ & $0.117 \pm 0.003$ & $0.01 \pm 0.05$ & $-0.32 \pm 0.07$ \\
T4 & $0.70 \pm 0.01$ & $0.116 \pm 0.002$ & $-0.05 \pm 0.06$ & $-0.32 \pm 0.06$ \\
T5 & $0.70 \pm 0.02$ & $0.130 \pm 0.003$ & $-0.04 \pm 0.06$ & $-0.38 \pm 0.05$ \\
T6 & $0.73 \pm 0.01$ & $0.121 \pm 0.003$ & $-0.09 \pm 0.04$ & $-0.37 \pm 0.06$ \\
T7 & $0.73 \pm 0.02$ & $0.126 \pm 0.005$ & $-0.09 \pm 0.07$ & $-0.34 \pm 0.17$ \\
T8 & $0.76 \pm 0.02$ & $0.122 \pm 0.003$ & $-0.16 \pm 0.08$ & $-0.33 \pm 0.25$ \\
T9 & $0.74 \pm 0.02$ & $0.124 \pm 0.005$ & $-0.17 \pm 0.04$ & $-0.35 \pm 0.06$ \\
T10 & $0.76 \pm 0.01$ & $0.135 \pm 0.009$ & $-0.13 \pm 0.08$ & $-0.36 \pm 0.18$ \\
T11 & $0.75 \pm 0.03$ & $0.132 \pm 0.006$ & $-0.11 \pm 0.06$ & $-0.38 \pm 0.05$ \\
T12 & $0.81 \pm 0.02$ & $0.135 \pm 0.005$ & $-0.23 \pm 0.07$ & $-0.36 \pm 0.05$ \\
\hline
\end{tabular}

bed arrangements. Most existing data sets of rough-bed flows are based on point measurements for a selected vertical, and therefore are not sufficient to characterize double-averaged (over space and time) flow properties [e.g., Cooper and Tait, 2010]. The experimental $p d f_{s}$ were then computed as the normalized discrete frequency distributions that account for both spatial and temporal variations. For each flow variable this frequency distribution was generated by clustering within constant-width bins the measurements acquired from the measurement area during a time interval containing 3000 frames (about $\Delta t=66.7$-s duration).

[10] A summary of mean and standard deviation of the streamwise velocity, $u_{f}$, measured at the laser level, $\mu_{u \text {, laser }}=$ $\mu_{u f}\left(z_{\text {laser }}\right)$ and $\sigma_{u, \text { laser }}=\sigma_{u f}\left(z_{\text {laser }}\right)$, respectively, are presented in Table 2 . Both parameters did not show any correlation with time, though weak variations were observed as the experiments proceeded. Values of the standard deviation were found to be slightly less than twice the shear velocity, $u_{s}$, which is consistent with measurements of time-averaged velocity moments observed in previous studies on near-bed turbulence in rough boundary open channels [e.g., Kironoto and Graf, 1994]. Figure 2a shows the experimental probability density function obtained for test T9 in a semi-log plot. The measured distributions extend over four to five orders of magnitude and hence they are suitable for investigating the deviation from the hypothesis of normality and the contributions from extreme events. The estimate of the skewness coefficient, $S_{\text {ulaser }}$, is reasonably close to zero and in most cases the variation of skewness and kurtosis, $K_{u \text {,laser }}$, with time falls within a narrow range. However, from Table 2 and Figure 2a distributions exhibit small but consistently negative excess kurtosis with an average value of -0.30 to -0.40 . The data also indicate a small negative skewness, resulting in negligible effects on the tails of the probability density function. In terms of shape, the distribution has a lower, wider peak around the mean and thinner tails than a normally distributed variable.

[11] The tendency to observe slightly sub-Gaussian statistics of the turbulent velocity fluctuations has been observed in the literature [e.g., Jiménez, 1998], although it is usually attributed to experimental uncertainty. The KolmogorovSmirnov and chi-square analyses performed on all the experimental tests have shown that the hypothesis of normality could be accepted at significance levels lower than $0.5 \%$. However, discrepancies from the Gaussian distribution
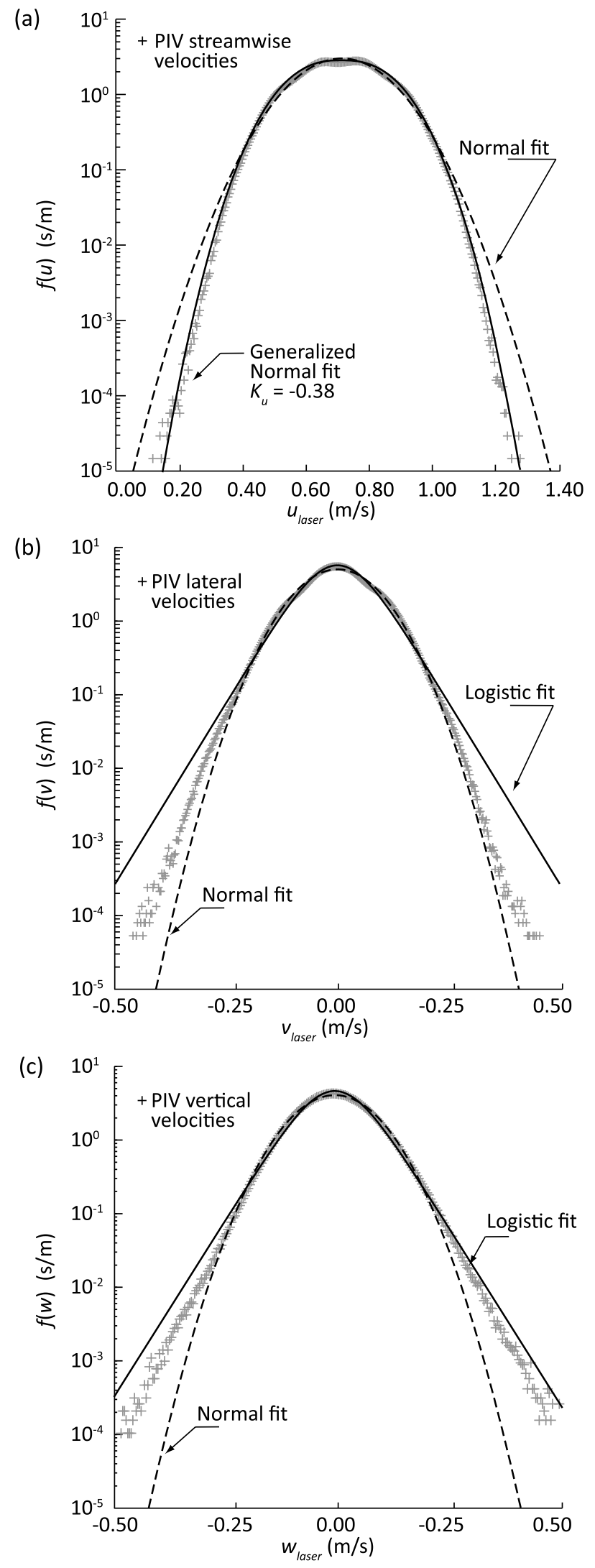

Figure 2. Frequency of (a) streamwise velocity, (b) lateral velocity and (c) vertical velocity for test T9. Solid lines represent generalized normal (or logistic) pdf; dotted lines represent Gaussian pdf. 
Table 3. Summary of Statistical Parameters for the Near-Bed Lateral and Vertical Velocities

\begin{tabular}{lccccrr}
\hline Test & $\sigma_{\mathrm{v}, \text { laser }}(\mathrm{m} / \mathrm{s})$ & $\mathrm{S}_{\mathrm{v}, \text { laser }}(-)$ & $\mathrm{K}_{\mathrm{v}, \text { laser }}(-)$ & $\sigma_{\mathrm{w}, \text { laser }}(\mathrm{m} / \mathrm{s})$ & $\mathrm{S}_{\mathrm{w}, \text { laser }}(-)$ & $\mathrm{K}_{\mathrm{w}, \text { laser }}(-)$ \\
\hline T1 & $0.074 \pm 0.002$ & $-0.04 \pm 0.07$ & $0.29 \pm 0.06$ & $0.081 \pm 0.003$ & $0.15 \pm 0.03$ & $1.71 \pm 1.02$ \\
T2 & $0.075 \pm 0.002$ & $0.01 \pm 0.02$ & $0.25 \pm 0.12$ & $0.091 \pm 0.003$ & $0.07 \pm 0.02$ & $1.44 \pm 0.64$ \\
T3 & $0.079 \pm 0.003$ & $0.00 \pm 0.04$ & $0.25 \pm 0.11$ & $0.102 \pm 0.002$ & $0.05 \pm 0.04$ & $1.53 \pm 0.38$ \\
T4 & $0.077 \pm 0.002$ & $0.00 \pm 0.03$ & $0.27 \pm 0.05$ & $0.097 \pm 0.003$ & $0.07 \pm 0.04$ & $1.42 \pm 0.41$ \\
T5 & $0.080 \pm 0.002$ & $0.00 \pm 0.06$ & $0.31 \pm 0.05$ & $0.104 \pm 0.003$ & $0.15 \pm 0.04$ & $1.94 \pm 0.56$ \\
T6 & $0.076 \pm 0.002$ & $0.05 \pm 0.03$ & $0.31 \pm 0.09$ & $0.098 \pm 0.002$ & $0.08 \pm 0.02$ & $1.17 \pm 0.18$ \\
T7 & $0.078 \pm 0.001$ & $0.02 \pm 0.05$ & $0.31 \pm 0.10$ & $0.098 \pm 0.004$ & $0.09 \pm 0.04$ & $1.26 \pm 0.75$ \\
T8 & $0.075 \pm 0.001$ & $-0.03 \pm 0.06$ & $0.32 \pm 0.09$ & $0.092 \pm 0.001$ & $0.13 \pm 0.02$ & $0.90 \pm 0.20$ \\
T9 & $0.078 \pm 0.003$ & $0.02 \pm 0.03$ & $0.29 \pm 0.06$ & $0.095 \pm 0.002$ & $0.11 \pm 0.02$ & $0.92 \pm 0.06$ \\
T10 & $0.083 \pm 0.005$ & $0.02 \pm 0.03$ & $0.30 \pm 0.06$ & $0.106 \pm 0.005$ & $0.13 \pm 0.02$ & $1.16 \pm 0.20$ \\
T11 & $0.079 \pm 0.004$ & $0.02 \pm 0.03$ & $0.29 \pm 0.07$ & $0.100 \pm 0.005$ & $0.14 \pm 0.04$ & $1.06 \pm 0.17$ \\
T12 & $0.077 \pm 0.002$ & $0.02 \pm 0.04$ & $0.30 \pm 0.06$ & $0.104 \pm 0.003$ & $0.11 \pm 0.04$ & $1.12 \pm 0.07$ \\
\hline
\end{tabular}

should be accounted for processes in which extreme values are important, such as sediment entrainment. Thus it is concluded that near-bed streamwise velocities can be reasonably considered to have a near-Gaussian distribution, which is reasonably approximated by a Generalized Normal model, i.e.

$$
\begin{aligned}
f_{U f}\left(u_{f} \mid \mu_{u f}, \sigma_{u f}, K_{u f}\right)= & \frac{\beta \cdot \Gamma^{1 / 2}(3 / \beta)}{2 \sigma_{u f} \cdot \Gamma^{3 / 2}(1 / \beta)} \\
& \cdot \exp \left\{-\left(\frac{\Gamma^{1 / 2}(3 / \beta)}{\Gamma^{1 / 2}(1 / \beta)} \cdot \frac{\left|u_{f}-\mu_{u f}\right|}{\sigma_{u f}}\right)^{\beta}\right\} \\
K_{u f}= & \frac{\Gamma(5 / \beta) \cdot \Gamma(1 / \beta)}{\Gamma^{3}(3 / \beta)}-3
\end{aligned}
$$

where $\Gamma(\beta)$ denotes the gamma function and $\beta$ is a shape parameter. This family of curves allows for distributions that have either longer tails than normal $(\beta<2)$ or a wider peak around the mean than normal $(\beta>2)$. From equation $(2 b)$ and allowing for $K_{u f}=K_{u, l a s e r}=-0.35$ one obtains $\beta=2.46$.

[12] Both the lateral and vertical velocity fluctuations deviate from Gaussian and are better approximated with leptokurtic distributions as shown in Figures $2 b$ and $2 c$ for test T9. The distribution of the vertical component, $w_{f}$, also demonstrates small deviation from symmetry, in agreement with past studies on Bagnold's theory linking sediment suspension with upward-directed residual Reynolds stress arising from asymmetrical shear turbulence [e.g., Leeder, 1983]. The standard deviation of the lateral component, $v_{f}$, was found to be approximately equal to the shear velocity, $u_{s}$, while the ratio $\left(\sigma_{v} / \sigma_{u}\right)_{\text {laser }}$ that characterizes the flow anisotropy is slightly less than the value 0.71 proposed by Nezu and Nakagawa [1993] for the relevant time-averaged distributions. The double-averaged turbulence intensity in the vertical direction appears slightly higher than the fluctuations of the lateral velocity. This is possibly due to the measurement error associated to the vertical velocity obtained with the stereoscopic PIV. The skewness and kurtosis factors for the lateral and vertical components are given in Table 3 . The vertical velocity shows the highest excess kurtosis with values ranging from 0.9 to 1.9. Figure 2 shows that the leptokurtic distributions have a more acute peak around the mean and longer tails. As a consequence using a Gaussian model to approximate the lateral and vertical velocity distributions may underestimate the probability of occurrence of a hydraulic event that causes entrainment.

\subsection{Drag Force on a Particle in a Fluid Flow}

[13] The distribution of the fluid shear stress is estimated from direct measurements of the local turbulent near-bed flow field. Schmeeckle et al. [2007] stated that the 'nominal drag', i.e., the actual force exerted by the fluid on a stationary particle, can be expressed as

$$
\begin{aligned}
F_{D}= & \frac{1}{2} \rho C_{D} A_{f} u_{f}\left(u_{f}^{2}+v_{f}^{2}+w_{f}^{2}\right)^{0.5} \\
& +\rho V_{s} C_{L}\left[v_{f}\left(\frac{\partial v_{f}}{\partial x}-\frac{\partial u_{f}}{\partial y}\right)+w_{f}\left(\frac{\partial w_{f}}{\partial x}-\frac{\partial u_{f}}{\partial z}\right)\right]+F_{A c c}
\end{aligned}
$$

where $A_{f}$ is the area of the particle exposed to the fluid; $V_{S}$ is the volume of the particle; $C_{D}$ and $C_{L}$ are the drag and lift coefficients, respectively. The first term in the right hand side of equation (3) is the hydrodynamic drag, which is dominant in steady one-dimensional flows. The acceleration force, $F_{A c c}$, is produced primarily by spatial pressure gradients and is usually difficult to estimate. The only components having nonzero mean are $u_{f}$ and $\partial u_{f} / \partial z$, other variables may become important in terms of their probability distribution. The standard deviation of the vertical component, $\sigma_{w}$, is about $10 \%$ of the mean streamwise velocity $\mu_{u}$. Neglecting $w_{f}$ in estimating the hydrodynamic drag results in an error within $2-3 \%$. Visual inspection of 2D contour plot velocity data (not reported) also indicates that for most of the time coherent flow structures could be observed that are larger than the particle size, suggesting that at the grain scale contributions due to lateral and streamwise gradients $\partial u_{f} / \partial x$ and $\partial u_{f} \partial y$ can be considered negligible. Schmeeckle et al. [2007] stated that the standard drag coefficient formulation can be used to predict reasonably well the instantaneous drag force from the instantaneous streamwise velocity. The drag coefficient was observed to be lower than the mean measured value for higher instantaneous velocities, approaching a maximum value as the velocity reduced to the lower range of the distribution. The resulting reduced drag variability with respect to the variability of $u_{f}\left|u_{f}\right|$ assuming constant $C_{D}$ was caused by neglecting the pressure term, $F_{A c c}$, and it was found to affect the downstream force by up to a maximum of $30 \%$. The use of the hydrodynamic drag as an approximation of equation (3) led to overestimating the real 
force on the particle when the near-bed downstream velocity is higher than the mean, i.e., for conditions at which a majority of the transport at the low sediment transport stages is expected to occur.

[14] In the literature the term shear stress usually refers to the space and time averaged fluid force exerted on an area of the bed. As sediments are actually entrained and moved by fluctuating forces, accounting for the limitations discussed above, the instantaneous fluid stress for an individual grain can be reasonably given by

$$
\tau_{f}=\frac{4 F_{D}}{\pi d^{2}} \cong \frac{1}{2} \rho C_{D} \cdot \omega_{f} \cdot u_{f}\left|u_{f}\right|
$$

where $\omega_{f}=A_{f} / A_{s}$ is the ratio of the exposed area to the crosssection of the particle, $A_{s}=\pi d^{2} / 4$, and $d$ is the particle size. The use of this approximation is supported by recent studies indicating that particle motion is highly correlated to the size of the near-bed streamwise velocity, and by experimental observations carried out by Tregnaghi et al. [2010]. They found that about $70 \%$ of the entrainment events were associated to peak values of the streamwise velocity.

\section{Scaling of the Turbulent Flow Field}

\subsection{Scaling of the Mean Velocity}

[15] Estimating the probability distribution of the fluid stress on the bed at a given time from flow velocity observations made above the bed surface and using equation (4) requires proper scaling equations to be identified. The probability density function of the streamwise velocity at an arbitrary distance from the bed, $u_{f}(z)$, can then be expressed in terms of the density function of the velocity at the laser level, $u_{f}\left(z_{\text {laser }}\right)$, which can be obtained from direct measurements.

[16] Previous studies on probability density functions of the velocity fluctuations in isotropic turbulence indicated a tendency toward universality, as they showed that the distribution of these quantities are independent of the wall normal distance at low Reynolds numbers [e.g., Balachandar and Sirovich, 1991]. Therefore, the standardized random variable $\left[u_{f}(z)-\mu_{u f}(z)\right] / \sigma_{u f}(z)$ can be described by the following distribution

$$
f_{U f}\left(\frac{u_{f}-\mu_{u f}}{\sigma_{u f}} \mid z\right)=f_{U f}\left(\frac{u_{f}-\mu_{u f}}{\sigma_{u f}}\right)
$$

which is independent of $z$. Subsequent observations carried out by Dinavahi et al. [1995] on the nature of the velocity fluctuations using data from direct numerical simulations extended these results outside the buffer layer of wallbounded turbulent flows. Equation (5) may hold also at the flow-sediment interface which is located within individual roughness elements to form the 'roughness layer' [Nikora et al., 2001]. The relationship between the probability density function of the streamwise velocity on a plane located at the distance $z$ from the bed, $u_{f}(z)$, and the density function of the velocity at the laser sheet elevation, $u_{f}\left(z_{\text {laser }}\right)$, can be reasonably approximated by using the relationships that link the relevant mean values and standard deviations at different distances from the bed surface.

[17] Nikora et al. [2004] showed that different specific expressions for $\mu_{u f}(z)$ within the roughness layer can be deduced using double-averaged equations. For flat sediment beds physical considerations suggest that the total drag term may monotonically increase toward the lower boundary of the roughness layer, leading to a linear velocity distribution within such layer. For the flow region above the roughness layer formulas for hydraulic resistance based on time-averaged equations are also valid for $\mu_{u f}(z)$. According to Nikora et al. [2001], the following equations are then considered to hold

$$
\begin{gathered}
\frac{\mu_{u f}(z)}{u_{s}}=\frac{1}{\kappa} \ln \left(\frac{z}{\delta_{R}}\right)+C_{1} \quad \text { for } z \geq \delta_{R} \\
\frac{\mu_{u f}(z)}{u_{s}}=C_{1} \frac{z}{\delta_{R}} \quad \text { for } 0 \leq z<\delta_{R}
\end{gathered}
$$

where $\kappa$ is the von Kármán constant; $\delta_{\mathrm{R}}$ is the depth of the flow roughness layer; $C_{1}$ is a coefficient related to the scale characterizing the flow dynamics below the roughness crest, which does not depend on the vertical coordinate $z$. The thickness of the roughness layer can be considered approximately equal to $4 \sigma_{z g}=1.0$ to $1.5 d_{50}$, with $\sigma_{z g}$ being the standard deviation of the bed elevations. For uniformly sized sediment (i.e., for $\sigma_{g}<1.35$ ) the roughness depth is expected to approach the lower values of the range, resulting in $\delta_{\mathrm{R}} \approx$ $d_{50}$. The coefficient $C_{1}$ is calibrated with the experimental data reported in Table 2 using equation (6a) and $z=z_{\text {laser }}$, as illustrated in Figure 3a. The value $C_{1}=7.1$ obtained from the data is in good agreement with results by Nikora et al. [2001] for spherical segment-type beds, although it is slightly higher than the range 5.5-6.0 found in previous studies on gravel bed roughness [e.g., Dittrich and Koll, 1997].

\subsection{Scaling of the Turbulence Intensity}

[18] Townsend [1976] argued that the relative turbulence intensity, i.e., the ratio of the standard deviation of the fluctuating velocities to the shear velocity, should be universal within the near-bed flow depth where the turbulent shear stress is approximately constant. Assuming that scaling laws developed for flows over fixed rough beds can be extended as a first approximation to weakly mobile-bed flows, the relationship of Kironoto and Graf [1994]

$$
\frac{\sigma_{u f}(z)}{u_{s}}=C_{2} \cdot \exp \left(-\frac{z}{h_{u}}\right)
$$

is used to evaluate the probability distribution of the streamwise velocity as a function of the wall-normal distance. The coefficient $C_{2}=1.8$ obtained from the current data (see Figure $3 \mathrm{~b}$ ) is slightly lower than the value $C_{2}=2.0$ proposed by Kironoto and Graf [1994]. From equations (6a), (6b), and (7) the following scaling relationships can therefore be obtained

$$
\begin{gathered}
\chi_{\mu}(z)=\frac{\mu_{u f}\left(z_{\text {laser }}\right)}{\mu_{u f}(z)}=\left[2.5 \ln \left(\frac{z_{\text {laser }}}{d}\right)+7.1\right] \cdot\left(\frac{d}{7.1 z}\right) \\
\chi_{\sigma}(z)=\frac{\sigma_{u f}\left(z_{\text {laser }}\right)}{\sigma_{u f}(z)}=\exp \left(\frac{z_{\text {laser }}-z}{h_{u}}\right)
\end{gathered}
$$



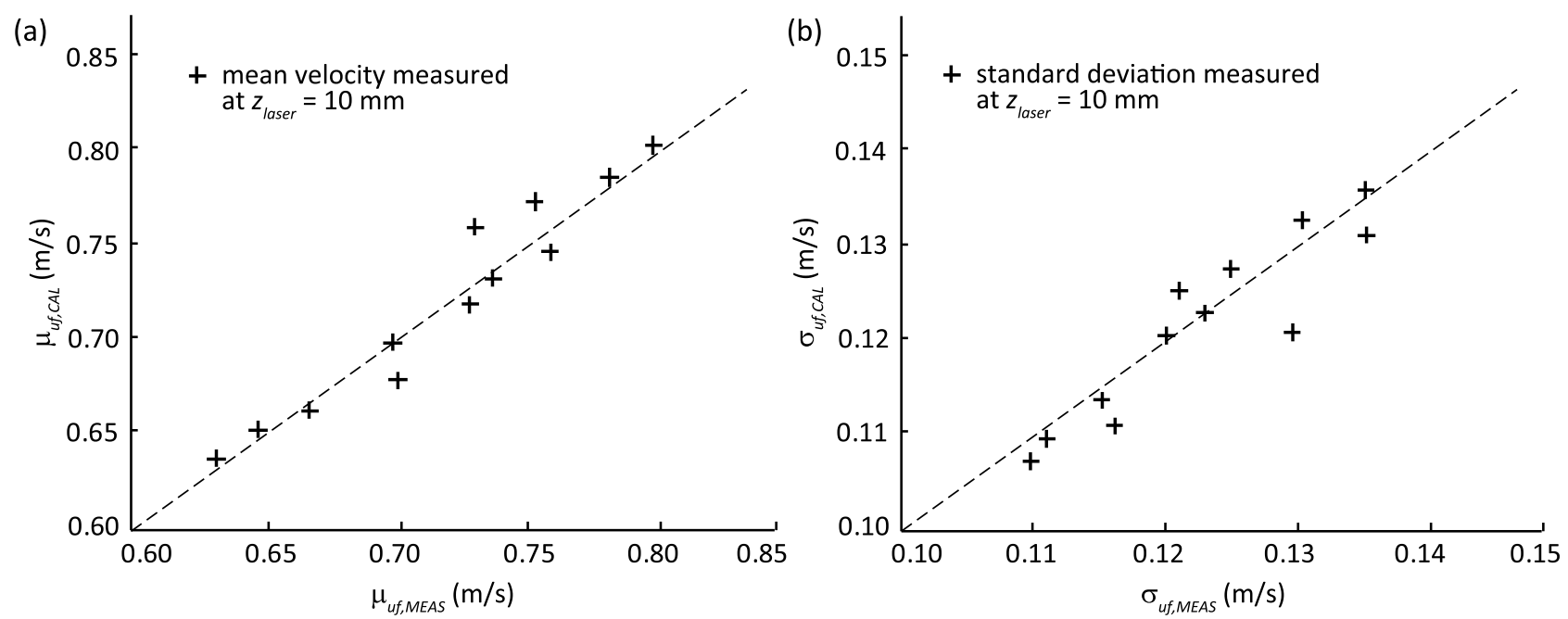

Figure 3. Calibration of experimental coefficients using data from Tests T1 to T12. (a) Mean velocity measured at the laser level. The coefficient $C_{1}=7.1$ is calibrated with the experimental data reported in Table 2 using equation (6a) and $z=z_{\text {laser }}$. (b) Standard deviation of measured velocities. The coefficient $C_{2}=1.8$ is obtained from the current data using (7) and $z=z_{\text {laser }}$.

These are used to estimate the experimental distribution of the fluid stress based on the information on the statistics of the velocity flow field measured above the sediment bed.

[19] Starred notation is used herein to denote dimensionless quantities. The scales that apply to fluid velocity and lengths are the shear velocity $u_{s}$ and the particle size $d$, respectively. Following the analysis developed in the companion paper [Tregnaghi et al., 2012] the conditional probability density function $f_{\mathrm{T} f}\left(\tau_{f}^{*} \mid z_{g}^{*}\right)$ is then given as parameter has been supported by systematic measurements of sediment bed micro-topography [Kirchner et al., 1990]. Resisting forces to grain motion should be represented by a distribution of values even for flat beds consisting of uniformly sized grains [McEwan and Heald, 2001]. This process is mainly governed by the relative grain projection above the mean bed, and the spatial variability of the 'pocket friction angle'. These variables are then considered as the key elements affect the stability of bed particles. Since it is

$$
f_{\mathrm{T} f}\left(\tau_{f}^{*} \mid z_{g}^{*}\right)= \begin{cases}\Gamma_{z}=\delta\left(z_{g}^{*}-\left(z_{\text {bed }}^{*}-\frac{1}{2}\right)\right) \cdot F_{Z g}\left(z_{\text {bed }}^{*}-\frac{1}{2}\right) & \text { for } z_{g}^{*} \leq\left(z_{\text {bed }}^{*}-\frac{1}{2}\right) \\ \frac{1}{\omega_{f} \sqrt{K\left|\tau_{f}^{*}\right|}} \cdot f_{U f}\left(\frac{\tau_{f}^{*}}{\left.\sqrt{K \omega_{f}\left|\tau_{f}^{*}\right|} \mid \frac{\mu_{u, \text { laser }}}{\chi_{\mu}\left(z_{f}^{*}\right)}, \frac{\sigma_{u, \text { laser }}}{\chi_{\sigma}^{2}\left(z_{f}^{*}\right)}, K_{u, \text { laser }}\right)}\right. & \text { for } z_{g}^{*}>\left(z_{\text {bed }}^{*}-\frac{1}{2}\right)\end{cases}
$$

where $K=0.5 \rho C_{D}\left(u_{s}\right)^{2} /(\gamma \Delta d)$ is a dimensionless coefficient, $\gamma$ the specific weight of water and $\Delta \approx 1.65$ the relative submerged density of sediments; $z_{b e d}^{*}$ is the top elevation of the particles composing the average bed surface; $z_{f}^{*}$ is the elevation of the application point of the drag force. Equation (9) can then be introduced in equation (1) to obtain the risk of entrainment, $R_{E}$. Such a relationship is obtained assuming that the density function of the streamwise velocity $f_{U f}$ follows a generalized normal distribution, and applying the fundamental theorem on transformation of random variables. In the next section, an experimental formulation of the joint density function $f_{\mathrm{T} g}, Z g$ will be provided based on DPM numerical simulations.

\section{DPM-Based Grain Resistance}

\subsection{Modeling Bed Resistance With Discrete Particle Models}

[20] The idea that the threshold shear stress for natural beds cannot be adequately described by a single-valued very difficult to obtain direct experimental measures of the critical shear stress distribution, friction angle measurements have been considered as a surrogate of the actual distribution of the stabilizing forces of bed particles. Analytical predictions of these variables were obtained only for simplified bed geometries. In most cases the variation around mean values of friction angles of natural sediments was treated in terms of measurement uncertainties and therefore averaged out.

[21] Discrete particle models can compute the force distribution for large numbers of surface particles. The forces that destabilize individual particles are then quantified directly rather than indirectly in the form of some measured flow parameter, thus conceptually providing a more realistic interpretation of the underlying physics of the grain resistance to motion. This modeling approach bears reasonable resemblance to the bed textures observed in gravel beds, as it reproduces the geometric variability inherent in a rough surface that controls the critical shear stress of each individual grain. Kirchner et al. [1990] reported that variability in friction angle measurements spread over similar ranges for both 

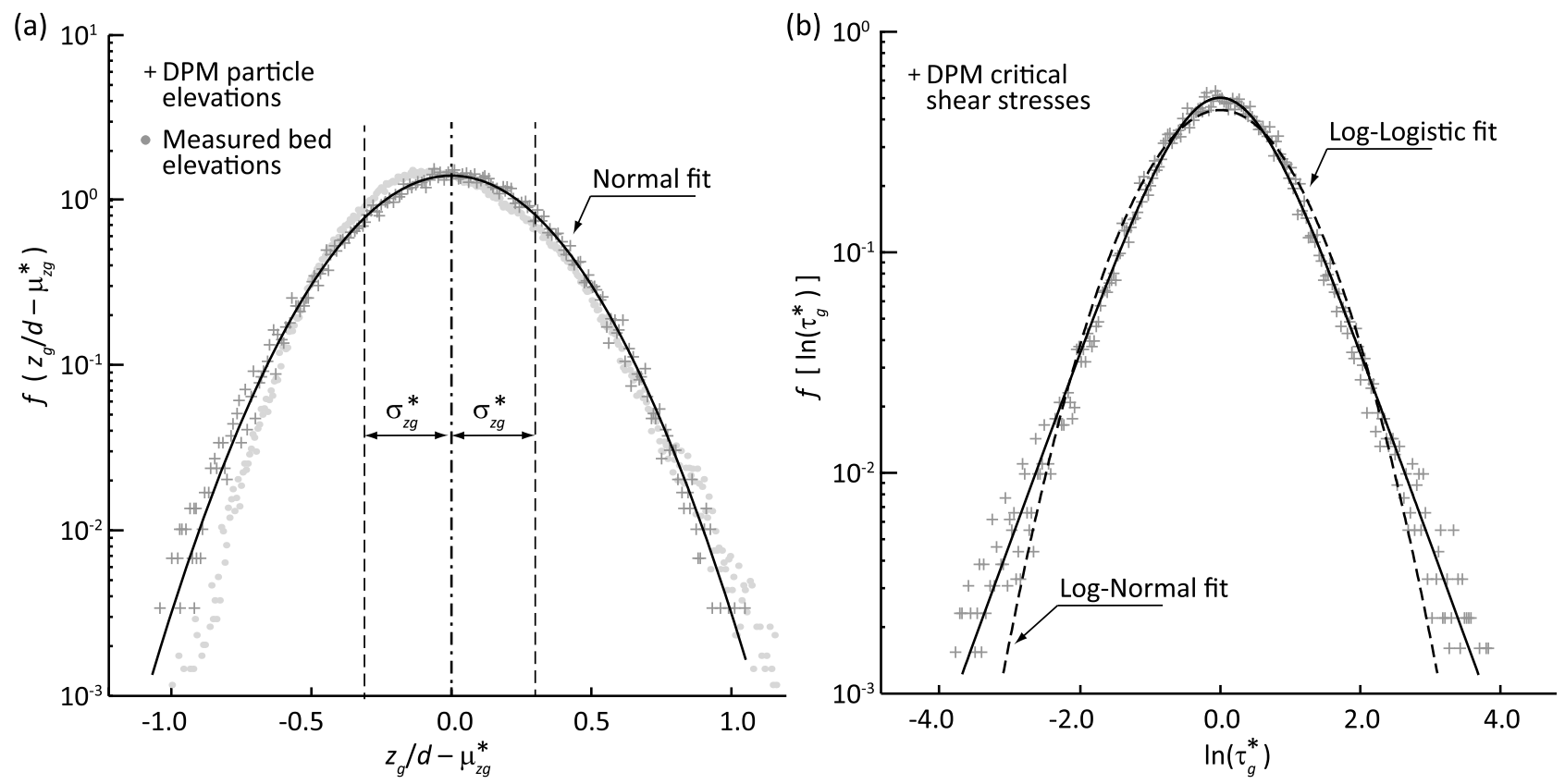

Figure 4. (a) Comparison of DPM numerical data and theoretical pdf of bed elevations. The numerical data closely follow a Gaussian model, with $\sigma_{z g}$ varying within the range $0.28 d$ to $0.30 d$. Laser scanner measurements also exhibit a near-Gaussian behavior. (b) Comparison of DPM numerical data and theoretical pdf of critical shear stresses. Simulated distributions indicate that the natural logarithmic of $\tau_{g}^{*}$ exhibits a nonzero excess kurtosis approximately equal to $1.20-1.35$.

natural and spherical test particles over rough beds. It is also intuitively obvious, but not clearly quantifiable, that such distributions are strongly dependent on the profile elevations of the bed surface. The degree of exposure of a particle to the flow contributes to the grain-scale variation in surface properties, this turns out to be one of the most important factors affecting grain stability. Numerical simulations were then performed to obtain the distribution of the resisting forces over the bed surface and their correlation to the exposures of the surface particles.

\subsection{Numerical Simulations}

[22] The discrete particle model originally developed by McEwan and Heald [2001] was used. The numerical bed was formed by releasing particles in sequence from a random position in a plane located well above the reference surface. The resulting sediment bed surface was defined as those grains that had no more than three grain to grain contact points (supporting grains) and were within a defined vertical distance of the mean bed level. The model calculates the critical value of the force acting in the streamwise direction, which produces a destabilizing moment sufficient to overcome the stabilizing moment due to the grain's submerged weight. No friction coefficient is considered in the moment analysis of grain stability. A grain is entrained through rotation about one of the three axes connecting the respective centers of its supporting grains. The value of the critical fluid force is obtained when the stabilizing moment is zero. Entrainment is assumed to occur about the axis for which the streamwise force magnitude is minimized. In the present numerical tests, a water-working module was used, which simulated a low uniform flow velocity during this pre-simulation phase, thus creating a more natural-like grain arrangement typical of water-worked beds.

[23] Figures $4 \mathrm{a}$ and $4 \mathrm{~b}$ show the surface elevation and threshold shear stress distributions, respectively, computed over a population of several thousand particles for 5-mm uniformly sized sediment beds and utilizing the sheltering effect module as described in McEwan and Heald [2001]. The distribution of bed elevations scaled with respect to the mean bed level and normalized to the sediment size was found to closely follow a Gaussian probabilistic model. The standard deviation $\sigma_{z g}$ of bed elevations varied within the range $0.28 d$ to $0.30 d$ for different tests, which matches well with values reported in the literature for natural sediment beds. This result is also consistent with the selected thickness of the roughness interface $\delta_{\mathrm{R}} \approx 4 \sigma_{z g} \approx d$ used to estimate the mean velocity vertical profile as discussed in the previous section. Bed elevation measurements were also performed with a laser scanner. These data were found to exhibit a near-Gaussian behavior and compare favorably with the numerical experiments (see Figure 4a). It is therefore concluded that the main textural properties of the artificial bed simulated by the discrete particle model reasonably approximate those of the sediment beds used in the flume experiments in the present study.

[24] For each particle comprising the bed surface the DPM calculates the critical value of the force acting in the streamwise direction, which produces a destabilizing moment sufficient to overcome the stabilizing moment due to the grain's submerged weight. Critical shear stress distributions were initially considered to be lognormal to a first approximation, however closer inspection of the simulated distributions has indicated that the natural $\operatorname{logarithmic}$ of $\tau_{\mathrm{g}}^{*}$ exhibits a nonzero 

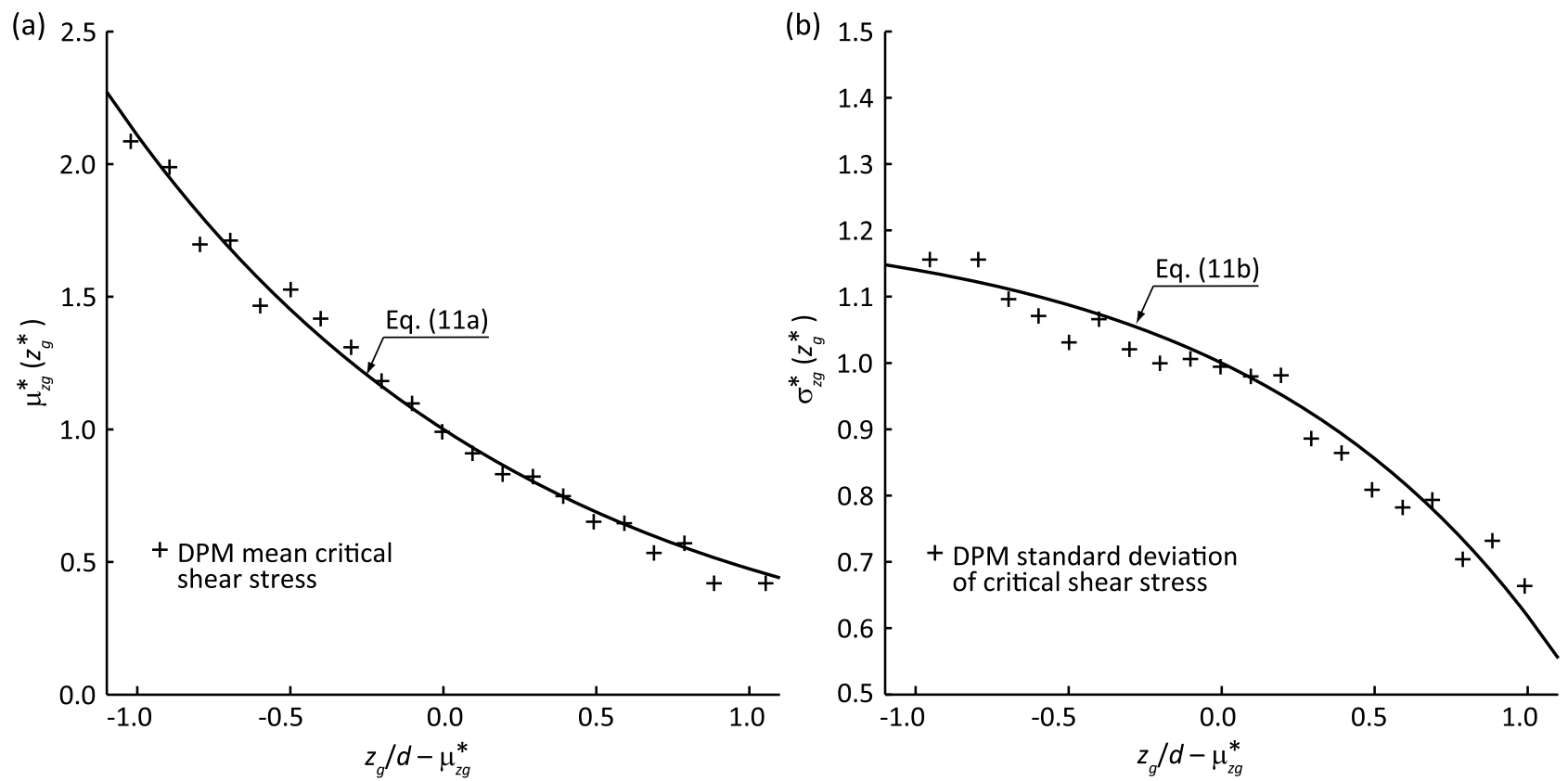

Figure 5. Geometric mean (a) and geometric standard deviation (b) of the critical shear stress distributions as a function of target grain elevation. Particles located approximately at the mean bed elevation exhibit the same distribution as the whole sample, i.e., the conditional density function of $\mathrm{T}_{g}^{*}$, given that $Z_{g}^{*}=\mu_{z g}^{*}$, is described by equation (10).

excess kurtosis approximately equal to $1.20-1.35$, although data are symmetrically distributed around their mean value. As a consequence, the numerical data are better approximated by a log-logistic model, i.e.

$$
\begin{aligned}
f_{T g}\left(\tau_{g}^{*} \mid \mu_{\tau g}^{*}, \sigma_{\tau g}^{*}\right)= & \frac{\pi}{4 \sqrt{3} \ln \left(\sigma_{\tau g}^{*}\right)} \cdot \frac{1}{\tau_{g}^{*}} \\
& \cdot \operatorname{sech}^{2}\left\{\frac{\pi}{2 \sqrt{3}} \cdot \frac{\ln \left(\tau_{g}^{*}\right)-\ln \left(\mu_{\tau g}^{*}\right)}{\ln \left(\sigma_{\tau g}^{*}\right)}\right\}
\end{aligned}
$$

where $\mu_{\tau g}^{*}$ and $\sigma_{\tau g}^{*}$ are the geometric mean and standard deviation of the dimensionless critical shear stress distribution. As shown in Figure 4b, the logistic density function has longer tails than the normal distribution and higher kurtosis, whose theoretical value is 1.2. The probabilistic models describing the surface elevation and threshold shear stress were not found to vary when increasing the uniform flow velocity from zero to about 3-4 times the shear velocity in the pre-simulation phase of the DPM. The geometric mean, $\mu_{\tau g}^{*}$, was observed to slightly increase from about 0.90 to 0.95 , while the standard deviation was found to be weakly dependent on water working conditions varying in the range $\sigma_{\tau g}^{*}=2.55$ to 2.65. The use of the water-working module removed the presence of very instable particles over the bed surface, thus slightly narrowing the distribution of bed elevations.

[25] The joint probability distribution of $\mathrm{T}_{g}^{*}$ and $Z_{g}^{*}$ was then estimated by splitting the whole sample of bed particles into a number of sub-samples with $z_{g}^{*}$ varying within a narrow range. The conditional density functions of $\mathrm{T}_{g}^{*}$, given the occurrence of $Z_{g}^{*}=z_{g}^{*}$, were found to follow approximately the same log-logistic model as the original numerical data set, with the scale and location parameters of the distribution depending on the particle elevation, i.e., $\mu_{\tau g}^{*}=$ $\mu_{\tau g}^{*}\left(z_{g}^{*}\right)$ and $\sigma_{\tau g}^{*}=\sigma_{\tau g}^{*}\left(z_{g}^{*}\right)$. Figures $5 \mathrm{a}$ and $5 \mathrm{~b}$ show the dependence on the particle elevation of the geometric mean and standard deviation, respectively, of the dimensionless critical shear stress, which can be expressed as follows

$$
\frac{\mu_{\tau g}^{*}\left(z_{g}^{*}\right)}{\mu_{\tau g}^{*}}=\mathrm{e}^{\alpha_{1}\left(z_{g}^{*}-\mu_{z g}^{*}\right)}
$$

$$
\frac{\sigma_{\tau g}^{*}\left(z_{g}^{*}\right)}{\sigma_{\tau g}^{*}}=1+\alpha_{2}\left(1-\mathrm{e}^{z_{g}^{*}-\mu_{z g}^{*}}\right)
$$

where $\alpha_{1} \approx-0.8$ and $\alpha_{2} \approx+0.2$ in the range $\mu_{z g}^{*}-1.0<z_{g}^{*}<$ $\mu_{z g}^{*}+1.0$ with coefficients of determination $\mathrm{R}^{2}=0.96$ and $\mathrm{R}^{2}=$ 0.92 , respectively. Note that particles located approximately at the mean bed elevation exhibit the same distribution as the whole sample, i.e., the conditional density function of $\mathrm{T}_{g}^{*}$, given that $Z_{g}^{*}=\mu_{z g}^{*}$, is described by equation (10). Given the density function of particle elevations, $f_{Z g}\left(z_{g}^{*}\right)$, combining equations (11a) and (11b) into equation (10) leads to the joint density function of $\mathrm{T}_{g}^{*}$ and $Z_{g}^{*}, f_{\mathrm{T} g Z_{g}}\left(\tau_{g}^{*}, z_{g}^{*}\right)$, which is defined as

$$
\begin{aligned}
f_{T g, Z g}\left(\tau_{g}^{*}, z_{g}^{*}\right) & =f_{T g}\left(\tau_{g}^{*} \mid z_{g}^{*}\right) \cdot f_{Z g}\left(z_{g}^{*}\right) \\
& =f_{T g}\left[\tau_{g}^{*} \mid \mu_{\tau g}^{*}\left(z_{g}^{*}\right), \sigma_{\tau g}^{*}\left(z_{g}^{*}\right)\right] \cdot f_{Z g}\left(z_{g}^{*}\right)
\end{aligned}
$$

Integrating equation (12) with respect to $\tau_{g}^{*}$ over the range $0<$ $\tau_{g}^{*}<\tau_{f}^{*}$ gives the partial derivative of the cumulative distribution function of $\mathrm{T}_{g}^{*}$ and $Z_{g}^{*}, \partial F_{\mathrm{T} g}, Z g / \partial z_{g}^{*}$, which has to be entered into equation (1) to provide the risk of entrainment given $R_{E}$. Figure 6 shows the comparison between the joint 

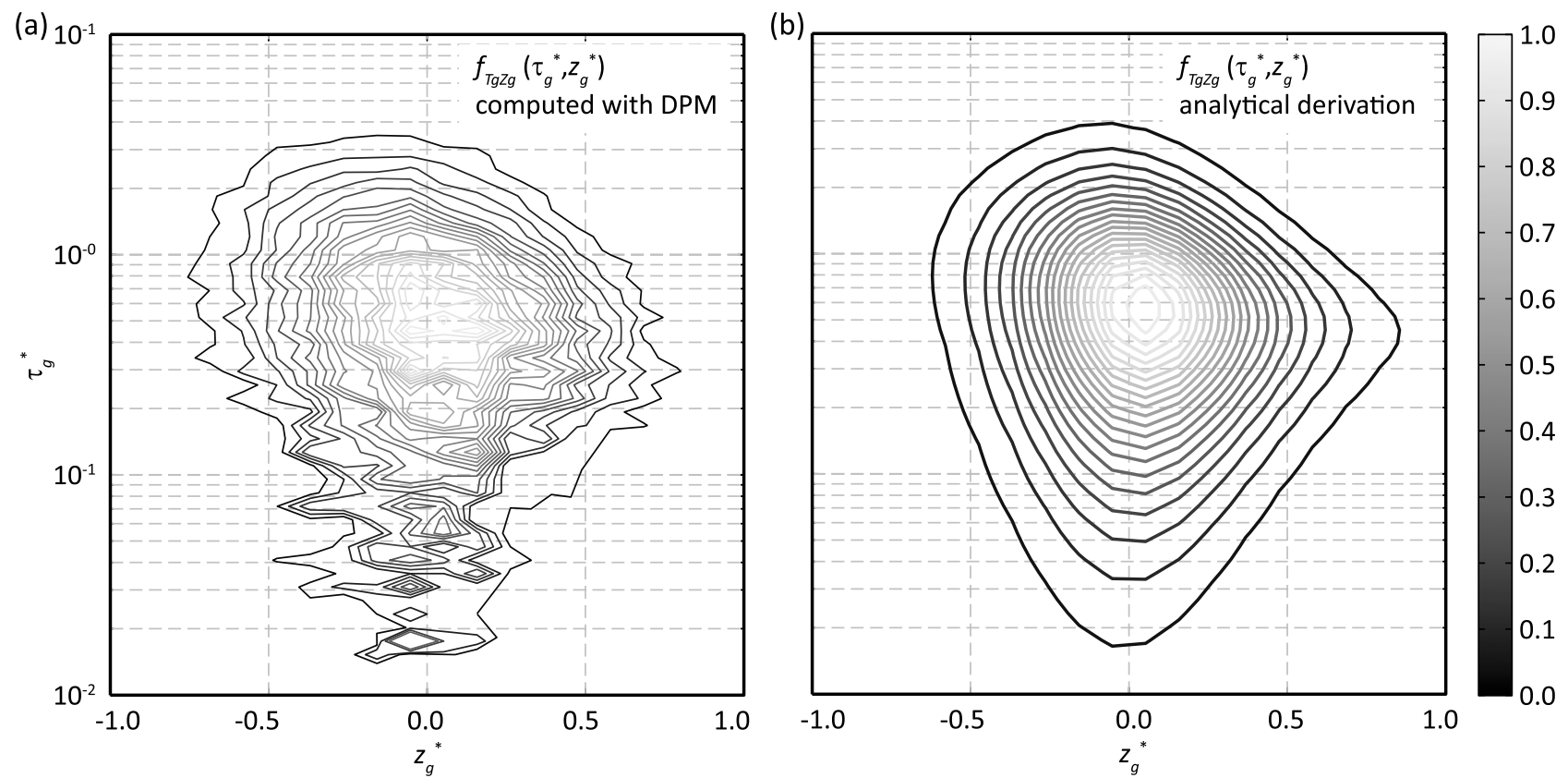

Figure 6. Comparison between joint density functions of $\mathrm{T}_{g}^{*}$ and $Z_{g}^{*}$ obtained from (a) DPM-based numerical simulations and (b) analytical derivation. The gray scale color map indicates the range of variation of $f_{\mathrm{T} g Z g}\left(\tau_{g}^{*}, z_{g}^{*}\right)$. This is obtained from the density function of particle elevations, $f_{Z g}\left(z_{g}^{*}\right)$, and combining equations (11a) and (11b) into equation (10).

density functions of $\mathrm{T}_{g}^{*}$ and $Z_{g}^{*}$ obtained from the numerical simulations discussed in this section and the analytical solution given by equation (12). To a first approximation, these data suggest that the joint distribution $\mathrm{T}_{g}^{*}$ and $Z_{g}^{*}$ can be obtained from the knowledge of the probabilistic model describing the distribution of $\mathrm{T}_{g}^{*}$ and the relationships that link mean and standard deviation of the critical shear stress to the vertical position of the bed particles. These data allows the theoretical probability of entrainment to be computed with equation (1) and then these results are compared to entrainment rate measurements collected from bed image analysis for tests T5, T9 and T11.

\section{Comparison of Experimental and Numerical Observations}

\subsection{Sensitivity Analysis of the Model}

[26] Model validation can be approached at two levels. Following a classical empirical approach the model can be compared against integrated physical quantities such as width/time averaged bed load transport rates. This integral approach often introduces calibration parameters with no clear physical meaning. True model validation should rather focus on the constituent sub-processes. This requires the collection of data able to describe individual processes at the grain scale.

[27] The model described by equations (1), (10) and (12) does not incorporate any specific coefficient that need integral sediment transport data for calibration, as most of the information required are obtained from direct observation of both experimental and numerical data at the grain scale. A number of physical parameters have to be selected that may introduce some uncertainty although few of them have been the subject of extensive discussion in the literature. These include 1) the position of the mean bed elevation, $\mu_{z g}=z_{\text {bed }}-$ $0.5 d$, with respect to the zero-velocity reference plane; 2 ) the standard deviation of bed elevations, which is linked to the thickness of the roughness interface according to the relation $4 \sigma_{z g}=\delta_{\mathrm{R}} ; 3$ ) the value of the drag coefficient $\left.C_{D} ; 4\right)$ and 5) the geometric mean and standard deviation, $\mu_{\tau g}^{*}$ and $\sigma_{\tau g}^{*}$ respectively, of the dimensionless critical shear stress distribution. Equation (1) has been computed using the following standard parameter values that have been argued to reasonably well approximate the physical conditions used in the experimental tests: $\mu_{z g}=-0.25 d, \sigma_{z g}=0.25 d, C_{D}=0.5$, $\mu_{\tau g}^{*}=0.95$ and $\sigma_{\tau g}^{*}=2.60$. A description follows on the influence of the variation in these parameters on the estimated risk of entrainment.

[28] Figure 7a shows the variation of $R_{E}$ as a function of the dimensionless mean bed elevation, with the standard deviation of bed elevations as a third parameter. The curves are computed for the hydraulic conditions of test T9 but similar trends can be obtained for different tests. For natural flat sediment beds the level of the top of the bed grains, $z_{\text {bed }}$, is commonly taken as $0.2-0.3 d$ above the zero-velocity reference plane, which closely approaches the mean surface elevation in a bed of uniformly sized spheres [e.g., Afzalimehr and Rennie, 2009]. The depth of the roughness, $\delta_{\mathrm{R}}$, may vary from at least $0.5 d$ to approximately $1.5 d$, resulting in $\sigma_{z g}=0.13 d$ to $0.37 d$, although such variation is expected to occur within a narrower range for uniform sediment, as suggested also by the results of the numerical experiments carried out with uniform spheres (see Figure 4a). Allowing for this evidence, if one selects a range of physical interest from Figure 7a it can be observed that $R_{E}$ varies from about $0.35 \%$ to $1.30 \%$, indicating a variation of almost four times within the lower and higher boundaries of the range. This theoretical observation, rather than being 

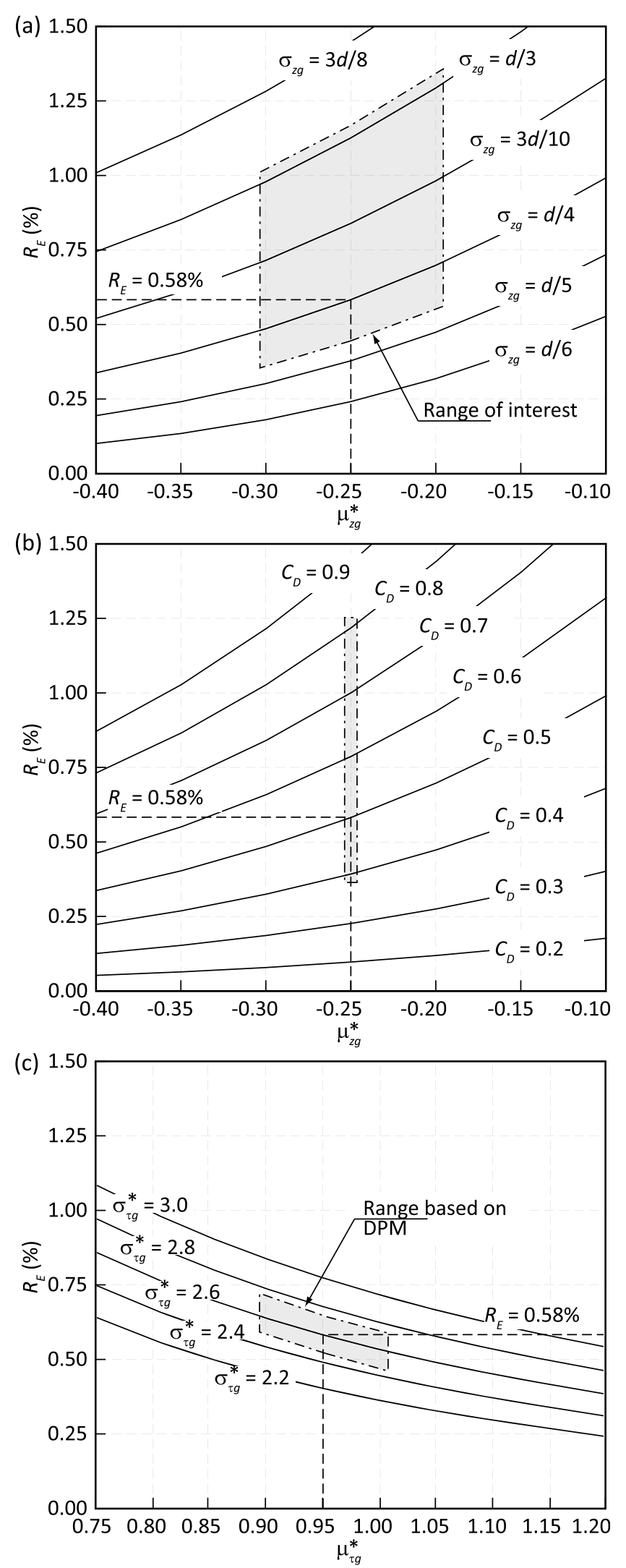

ascribed to the limitation of the model, stems from the effect of the natural variability in particle exposure to the flow in sediment deposits on the entrainment process, and it is likely to be partly responsible for the discrepancies observed in sediment transport data measured at low transport stages even for very similar hydraulic conditions.

[29] Figure 7b shows the dependency of $R_{E}$ on the drag coefficient as the mean bed elevation varies from $\mu_{z g}=-0.4 d$ to $-0.1 d$. The estimated value of the drag coefficient is one of the major sources of uncertainty in models that relate fluid forces to near-bed flow velocities. Currently it is commonly accepted that the drag coefficient can be approximated by that of a sphere settling in still water at its terminal settling velocity, i.e., $C_{D}=0.4$. Recently, experimental work by Schmeeckle et al. [2007] reported that the average drag coefficient can be assumed $C_{D}=0.7$ to 0.9 for particles of different shapes, although for the highest fluctuations above the mean, i.e., for the fluctuations that generally are more important for determining sediment transport, the instantaneous $C_{D}$ was found to asymptotically approach about 0.4 to 0.5 . As shown in Figure $7 \mathrm{~b}$, for $\mu_{z g}=-0.25 d$ the risk of entrainment varies from about $R_{E}=0.40 \%$ for $C_{D}=0.4$ to $R_{E}=1.20 \%$ for $C_{D}=$ 0.8 , and the influence of the selected coefficient becomes more important as the particle protrusion within the fluid increases. In this case, the variability of the results is linked to the choice of the parameterization of fluid stresses given in equation (4), which possibly requires further theoretical and experimental improvement, unless accurate estimates of the drag coefficient in such geometric configurations can be obtained.

[30] To a lesser extent the estimation of $R_{E}$ is also dependent on the choice of first and second order moments of the dimensionless critical shear stress distribution. Although many studies have recognized that the resisting forces to grain motion should be represented by a distribution of values, determining such distribution experimentally is likely to be very difficult. To our knowledge few studies are available [Kirchner et al., 1990; Cheng et al., 2003] that provided a theoretical derivation of the variation of the critical shear stress based on measurements of bed particle arrangements on a flat bed. Bottacin-Busolin et al. [2008] demonstrated that the probability distribution of individual grain resistance could be measured from the statistics of the near-bed velocity field and of sediment entrainment, however they obtained only a small part of the total resisting forces curve, as flow conditions were just above the critical threshold for sediment motion. To date DPM modeling possibly provides the only practical means to evaluate these distributions, although the numerical results are limited in different ways due to the idealization of the simulation. Figure $7 \mathrm{c}$ shows the variation

Figure 7. Sensitivity analysis of the model. (a) Entrainment risk as a function of $\mu_{z g} / d$ and $\sigma_{z g} / d$. Within the a range of physical interest (shaded area) $R_{E}$ varies from about $0.35 \%$ to $1.30 \%$, which is linked to the variability of particle exposures in natural sediment beds. (b) Entrainment risk as a function of $\mu_{z g} / d$ and $C_{D}$. For $\mu_{z g}=-0.25 d R_{E}$ varies from about $0.40 \% C_{D}=0.4$ to $1.20 \%$ for $C_{D}=0.8$ and the influence of the selected coefficient becomes more important as the particle protrusion within the fluid increases. (c) Entrainment risk as a function of $\mu_{\tau g}^{*}$ and $\sigma_{\tau g}^{*}$. The shaded area indicates the range of possible outcomes form the numerical simulations. 
Table 4. Summary of Results

\begin{tabular}{lccc}
\hline \multicolumn{1}{c}{ Test } & T5 & T9 & T11 \\
\hline $\bar{E}_{s}\left(\mathrm{~g} / \mathrm{m}^{2} \mathrm{~s}\right)$ & 49.3 & 56.2 & 65.4 \\
$\bar{R}_{E}(\%)$ & 0.47 & 0.56 & 0.61 \\
\hline
\end{tabular}

of $R_{E}$ as a function of the geometric mean and standard deviation of the dimensionless critical shear stress, indicating that the variability of the entrainment risk with the range of possible outcomes form the numerical simulation falls within a narrow band.

\subsection{Entrainment Risk and Entrainment Rate}

[31] The sediment transport rate has been observed to be highly fluctuating even for steady flows. After having examined the response of the model to the choice of some important physical parameters, the underlying equations are tested against the capability to reproduce this significant aspect of bed load at low transport stages. The original study by Einstein [1942] stated that

$$
E_{s} \propto n_{s} \cdot R_{E}
$$

where $E_{s}$ is the entrainment rate per unit time and over a unit area, and $n_{s}$ is the number of available sediment particles distributed over the bed surface. If one assumes that the spatial characteristics of the turbulent flow field are frozen during the time of sampling, from equation (1) a single value of $R_{E}$ is obtained for each experiment. However, it is expected that at the scale of investigation irregularities of the flow field occur even in controlled experimental conditions. In this regard, Tregnaghi et al. [2010] analyzed the temporal trend of the near-bed flow fields measured in the experiments described in this study and reported that spatially distributed flow structures are larger than the typical grain size and produced alternate regions of low and high flow velocities.

[32] Using the measured flow evidence, the temporal variation of $R_{E}$ has been obtained from equation (1) evaluating the statistics of the velocity distribution over a time interval $\Delta t=1 \mathrm{~s}$. Results summarized in Table 4 show that the averaged quantities over the period of observation, $\bar{E}_{S}$ and $\bar{R}_{E}$, increase for test T5 to test T11 with an approximately constant ratio as predicted by equation (13). Figures $8 \mathrm{a}-8 \mathrm{c}$ indicate, for tests T5, T9 and T11 respectively, that the predicted time-varying entrainment risk reasonably replicates the fluctuations of the measured entrainment rate, as crests and troughs of both time series are found to be well correlated. Figures $8 \mathrm{~d}-8 \mathrm{f}$ show the temporal trends of the spatialaveraged flow velocity and standard deviation $\mu_{u f}(t)$ and $\sigma_{u f}(t)$, scaled to the double-averaged flow velocity and standard deviation $\mu_{u f}$ and $\sigma_{u f}$ estimated over the period of observation. From comparison with $E_{s}(t) / \bar{E}_{s}$, it can be seen that the variability in entrainment appears to be largely driven by differences in the turbulence structure of the flow. At least three spatial flow patterns can be identified that are linked to large values of the entrainment rate, which include uniformly distributed high-velocity structures with relatively lowfrequency $(\mathrm{A} 1, \mathrm{~B} 1$ and $\mathrm{C} 1)$, smaller structures with alternate peak and low velocities (A2, B2 and C2), and more complex patterns presenting both high mean velocity and large fluctuations (A3, B3 and C3). It can be suggested that a variety of flow patterns may lead to comparable sediment transport fluctuations, as they are characterized by equivalent risks of entrainment. The three entraining spatial patterns all exhibit the presence of high velocity flow structures, however they affect the entrainment mechanism in different ways depending on the degree of particle exposure, as illustrated in the following section.

[33] The ratios of the instantaneous entrainment rate to its temporal average, $E_{S}(t) / \bar{E}_{S}$, appear larger than the relevant ratios of the entrainment risk, $R_{E}(t) / \bar{R}_{E}$. Such discrepancy may be partly due to an additional entrainment process not described by equation (1). As demonstrated by Ancey et al. [2008] this depends on the number of moving particles. The difference may therefore reflect the additional motion of bed particles owing to collisions with other particles and to the potential occurrence of collective entrainment. One other source of uncertainty is represented by the parameterization of fluid stresses as a function of the streamwise velocity only. The vertical velocity, which may be important for lift force, was not accounted for as its effect on the entrainment of exposed particles is reasonably negligible. This contribution may be up to $10 \%$ of the total entrainment probability for partially sheltered particles [Papanicolaou et al., 2002]. In the next section it is demonstrated that these particles are more sensitive to local modifications of the near-bed flow structure, therefore the additional effect of the vertical velocity may have some significance in explaining the observed oscillations of the entrainment rate around the mean value. It is concluded that the prediction of the entrainment risk, $R_{E}$, may be only slightly underestimated in terms of temporal average over a relatively long period. Further modeling improvements are required to predict the occurrence of larger entrainment pulsations.

\subsection{Selective Entrainment of Particles}

[34] Following Bottacin-Busolin et al. [2008], the risk density function $r_{\mathrm{E}}$ can be expressed as

$$
r_{E}\left(\tau_{f}^{*}, z_{g}^{*}\right)=f_{\mathrm{T} f}\left(\tau_{f}^{*} \mid z_{g}^{*}\right) \cdot \frac{\partial}{\partial z_{g}^{*}} F_{\mathrm{T} g, Z g}\left(\tau_{f}^{*}, z_{g}^{*}\right)
$$

where the elemental risk $r_{\mathrm{E}} \cdot \delta \tau_{f}^{*}$ gives the fraction of grains per unit area moved by a fluid stress lying in the range $\tau_{f}^{*} \pm$ $\delta \tau_{f}^{* / 2}$ and at a specific location $z_{g}$. Integrating equation (14) with respect to $\tau_{f}^{*}$ and multiplying by $f_{Z g}\left(z_{g}^{*}\right)$ leads to the marginal entrainment risk $R_{\mathrm{E}}\left(z_{g}^{*}\right)$. This represents the probability of entrainment of particles located at the position $z_{g}^{*}$, weighed by the relative frequency of occurrence of the given particle fraction.

[35] Figure 9a shows the marginal risk of entrainment $R_{\mathrm{E}}\left(z_{g}^{*}\right)$ as a function of the velocity mean, $\mu_{u f}$, and standard deviation, $\sigma_{u f}$, for a fully exposed particle and for flow conditions relevant to test $\mathrm{T} 9$. The filled area approximates the range of long-term disturbances of the flow field that were observed during the experiment (see Figure 8e). Points (1) and (3) represent temporal reductions of the velocity mean and standard deviation, respectively. They are ' $R_{\mathrm{E}}$-equivalent' as they give rise to identical attenuation of the entrainment risk $R_{\mathrm{E}}$, i.e., the average probability associated to the whole sediment population. However, different variations of the marginal risk $R_{\mathrm{E}}\left(z_{g}^{*}\right)$ apply for particles located at different positions. Similarly, points (2) and (4) identify flow conditions where the 

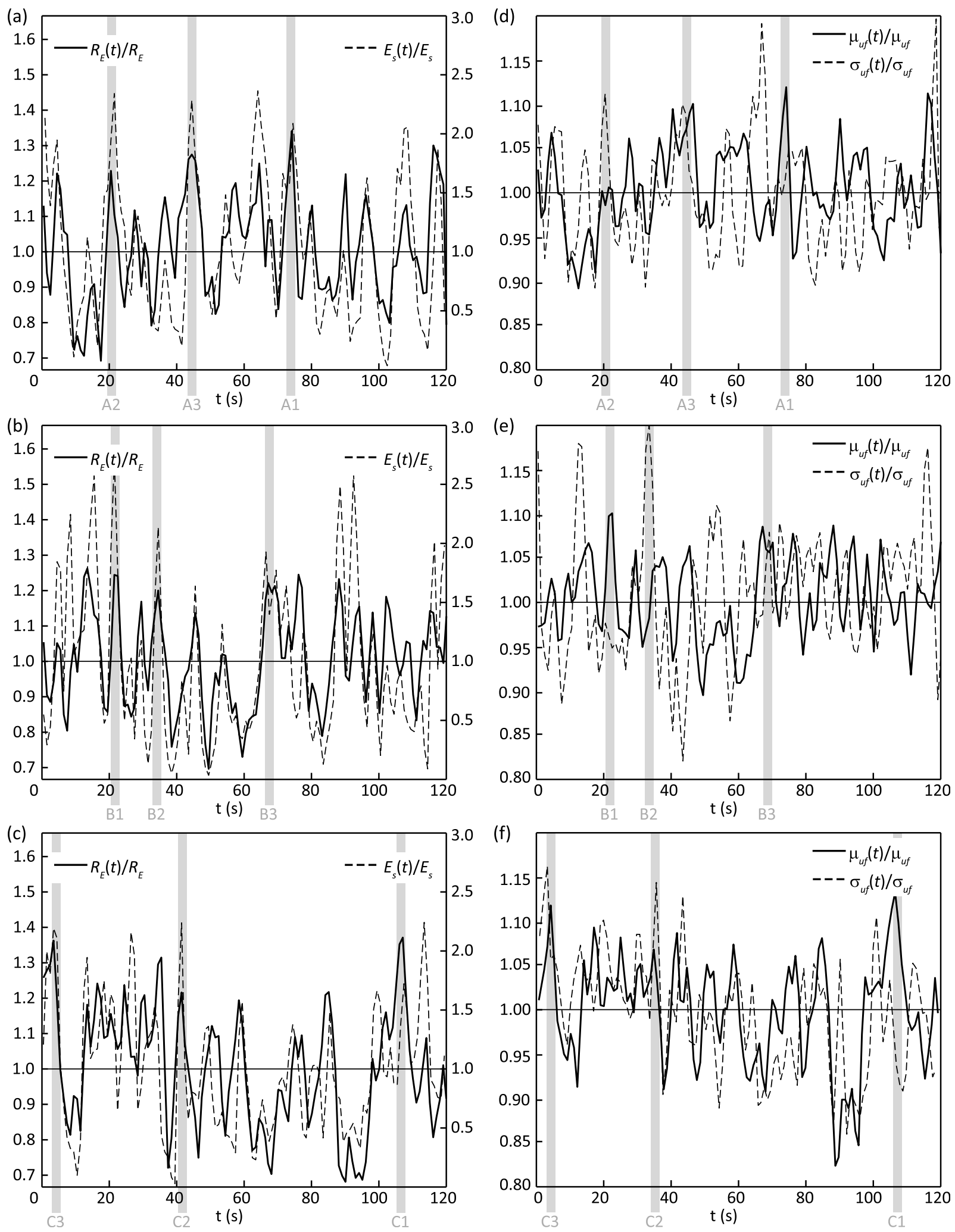

Figure 8. $(\mathrm{a}-\mathrm{c})$ Time-varying entrainment risk compared with fluctuations of measured entrainment rate. $(\mathrm{d}-\mathrm{f})$ This evidence is shown to be linked to spatial patterns of the turbulent flow structure. At least three spatial flow patterns can be identified that are linked to large values of the entrainment rate, which include uniformly distributed high-velocity structures with relatively low-frequency (A1, B1 and C1), smaller structures with alternate peak and low velocities (A2, B2 and C2), and more complex patterns presenting both high mean velocity and large fluctuations (A3, B3 and C3). 


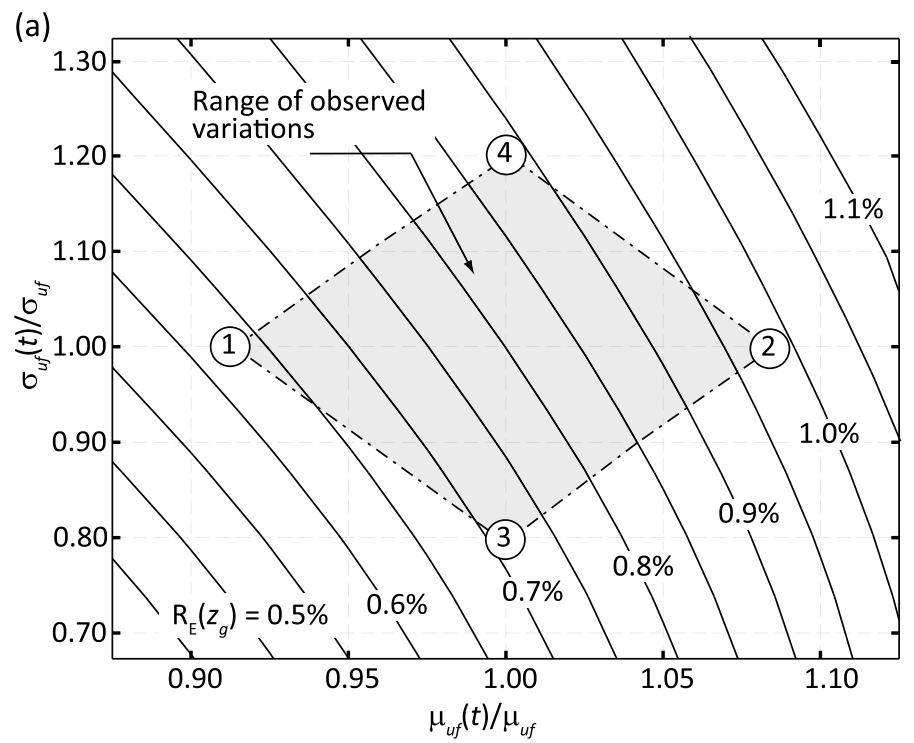

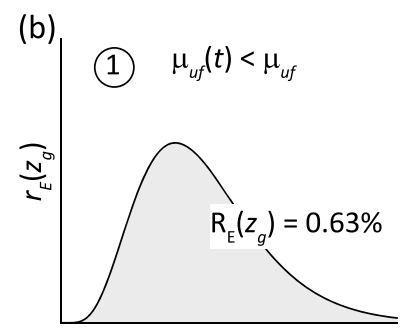
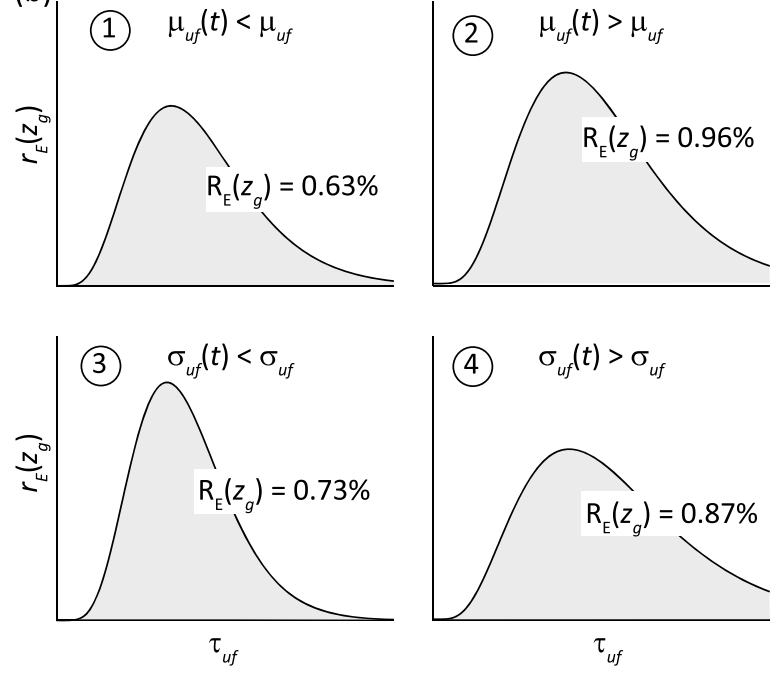

Figure 9. (a) Marginal risk of entrainment for a fully exposed particle as a function of velocity mean and standard deviation. (b) Density risk computed for different spatial patterns of the turbulent flow structure. It is shown that for a fully exposed particle variations of the averaged velocity are more effective for particle entrainment.

moments of the velocity distribution increase resulting in comparable increments of $R_{\mathrm{E}}$. From Figure $9 \mathrm{~b}$, it can be drawn that positive variations of $\mu_{u f}$ are more effective in determining the initiation of motion of the more exposed particles compared to $R_{\mathrm{E}}$-equivalent variations of the $\sigma_{u f}$. Comparing the transitions from state (1) to (2) and from state (3) to (4), respectively, shows that the relative increment of the marginal risk $R_{\mathrm{E}}\left(z_{g}^{*}\right)$ is about twice in the former case.

[36] Figure 10a shows similar results for a particle that is partially sheltered. By comparison with Figure 9a, it is immediately apparent that the direction of maximum increase of $R_{\mathrm{E}}\left(z_{\mathrm{g}}^{*}\right)$ is rotated, which indicates that in this case variations of the turbulence intensity are mostly effective in determining the dislodgement of particles. Exposed particles mainly contribute to the entrainment rate for all examined flow conditions, although they are relatively less frequent over the bed surface. However, particles that are partially sheltered are more sensitive to long-term fluctuations of the near-bed flow structure. Figure $10 \mathrm{~b}$ show that variations of the $\sigma_{u f}$ may induce up to fivefold increases in the marginal risk $R_{\mathrm{E}}\left(z_{g}^{*}\right)$ of
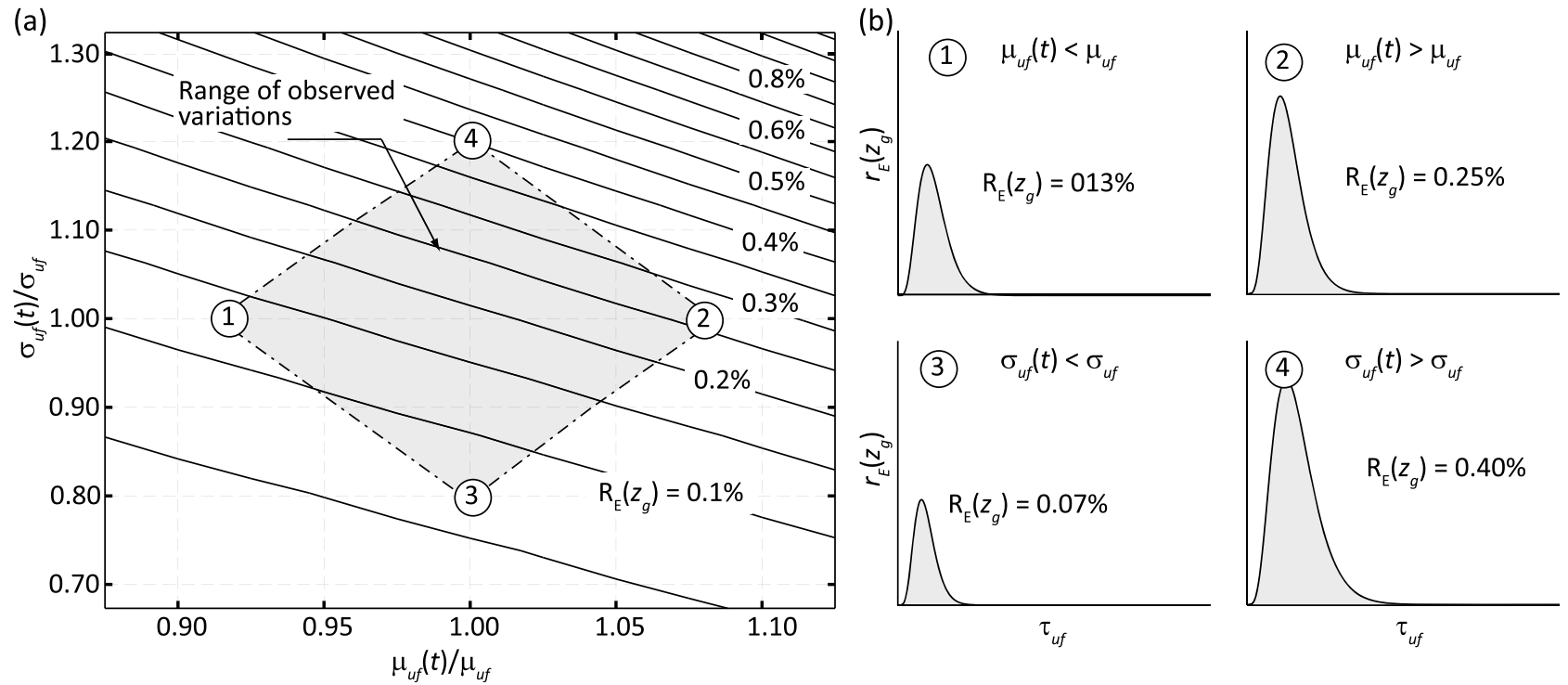

Figure 10. (a) Marginal risk of entrainment for a partially sheltered particle as a function of velocity mean and standard deviation. (b) Density risk computed for different spatial patterns of the turbulent flow structure. It is shown that for a partially sheltered exposed particle variations of the turbulence intensity are more effective for particle entrainment. 
partially sheltered particles, while $R_{\mathrm{E}}$-equivalent variations of $\mu_{u f}$ cause at most a $30 \%$ increase of the marginal risk $R_{\mathrm{E}}\left(z_{g}^{*}\right)$ for exposed particles. This follows from fully exposed particles being subject to relatively high velocities for most of the time. As a consequence, modifications of the turbulent flow field induce relatively small incremental overlapping between the marginal distributions of the fluid and critical stresses [see Tregnaghi et al., 2012, Figure 5]. On the other hand, partially sheltered particles rarely experience forces that can cause the dislodging from their pocket positions. This results in significant increments of the overlapping between the marginal stress distributions when slight variations of the averaged local flow field occur. Based on a similar schematic, it can be drawn that $\sigma_{u f}$ primarily controls the entraining of partially sheltered particles as the relative turbulence intensity, i.e., the ratio of $\sigma_{u f}$ to $\mu_{u f}$, reduces with the particle elevation $z_{g}$, thus rendering the $\mu_{u f}$ based mechanism mostly effective for fully exposed particles.

[37] It is concluded that although local changes of the near-bed flow structure are equivalent in terms of the mean entrainment probability $R_{\mathrm{E}}$ associated to the whole sediment populations, they are selective in terms of the marginal probability $R_{\mathrm{E}}\left(z_{g}^{*}\right)$ for individual particles located at different positions. These insights have some significance for the study of the sediment dynamics at the fundamental scale, i.e., the grain scale, by sustaining the possibility of investigating the Lagrangian motion of individual particles in complex fluid flows.

\section{Conclusions}

[38] Despite substantial progress made over the last two decades in the physical understanding of the sediment transport, the ability to predict transport rate in rivers remains poor. This can be ascribed to the structure of models not reflecting the underlying physical processes. In this study incipient motion is mathematically described as a random process that accounts for flow turbulence fluctuations and the effects of local surface heterogeneities. This study aimed to test the probabilistic modeling approach applied to the entrainment and transport processes by using high resolution data of near bed velocity and grain motion. The current modeling approach differed from previously developed stochastic models by including the statistical dependence of the fluid forces acting on the static bed particles and the critical forces required to begin motion, and by linking these forces to the probabilistic distribution of the grain exposure.

[39] A reasonable reproduction of the pulsating sediment entrainment process observed in flat gravel beds has been achieved. The analysis of the model equations has shown that there is a strong link between the critical shear stress required for entrainment and the statistical description of the bed elevations. The choice of the position of the mean bed level with respect to the vertical profile of the streamwise velocity and the depth of the roughness layer was also found to effect significantly the estimated probability of entrainment. This evidence demonstrates that gravel bed streams having similar depth averaged hydraulic conditions may have significantly different sediment transport rates even if the sediment displays a similar surface particle size distribution. Instantaneous fluctuations of the entrainment rate were found to correlate well with the temporal variability of the near bed fluid velocities. By comparing the temporal sequence of entrainment events with the relevant time-varying entrainment risk, a number of specific spatial flow structures were identified that caused large fluctuations of the entrainment rate.

[40] The entraining spatial patterns all exhibit the presence of high velocity flow structures, however they affect the entrainment mechanism in different ways. It was found that temporal variations of the near-bed velocity distribution may be equivalent in terms of the mean entrainment probability $R_{\mathrm{E}}$ associated to the whole sediment populations. However, they are selective in terms of the marginal probability $R_{\mathrm{E}}\left(z_{g}^{*}\right)$, which identifies the entrainment probability of individual particles located at different positions. This selective mechanism was demonstrated to be a direct consequence of the different interaction between the near-bed flow and the resistance of particle having variable degree of exposure. Such interaction was then explained in light of the theoretical framework underlying equation (1), as illustrated in the relevant companion paper [Tregnaghi et al., 2012]. These new insight should motivate further research on the motion of individual particles in complex fluid flows, with particular reference on how sediment dynamics affect the transport of particulate matter in rivers.

[41] It is concluded that a probabilistically based method to predict the temporal pattern of sediment entrainment is possible, but that detailed information of the bed surface and an accurate statistical description of the near bed flow is needed. In a steady uniform boundary layer the turbulent fluctuations scale with the shear velocity, bed surface elevations can also be approximated by simple probabilistic functions and so it is suggested that the methods discussed in this study can be used to estimate pulsating entrainment and transport. In nonuniform or unsteady flows the knowledge of the near-bed turbulence structure would need to be improved. However, the coupling between near-bed flow and the probabilistic risk of entrainment in non-uniform or unsteady flows is expected to be modeled with similar equations to those developed for steady flow conditions.

\section{Notation}

$$
\begin{aligned}
& A_{f} \text { particle area exposed to the fluid, }\left[\mathrm{L}^{2}\right] \text {. } \\
& A_{s} \text { particle area, }\left[\mathrm{L}^{2}\right] \text {. } \\
& E_{s} \text { entrainment rate, }\left[\mathrm{ML}^{-2} \mathrm{~T}^{-1}\right] \text {. } \\
& \bar{E}_{s} \text { temporally averaged entrainment rate, } \\
& {\left[\mathrm{ML}^{-2} \mathrm{~T}^{-1}\right] \text {. }} \\
& F_{D} \text { drag force, }\left[\mathrm{MLT}^{-2}\right] \text {. } \\
& F_{Z g} \text { cumulative distribution function of } z_{g} \text {. } \\
& F_{\mathrm{Tg}}, \mathrm{Zg} \text { joint cumulative distribution function of } \\
& \tau_{g} \text { and } z_{g} \text {. } \\
& K_{u}, K_{v}, K_{w} \text { kurtosis coefficient of } u_{f}, v_{f}, w_{f} \text {. } \\
& Q \text { water discharge, }\left[\mathrm{L}^{3} \mathrm{~T}^{-1}\right] \text {. } \\
& \left.Q_{s} \text { solid discharge, [MT }{ }^{-1}\right] \text {. } \\
& R_{e} \text { Reynolds number. } \\
& R_{E} \text { risk of entrainment. } \\
& \bar{R}_{E} \text { temporal average risk of entrainment } \\
& R_{H} \text { hydraulic radius, [L]. } \\
& S \text { bed slope. } \\
& S_{u}, S_{v}, S_{w} \text { skewness coefficient of } u_{f}, v_{f}, w_{f} \text {. } \\
& \left.U \text { mean flow velocity, [ } \mathrm{LT}^{-1}\right] \text {. }
\end{aligned}
$$


$V_{s}$ particle volume, $\left[\mathrm{L}^{3}\right]$.

$d$ representative grain size of the sediment, [L].

$d_{50}$ mean grain size of the sediment distribution, [L].

$g$ acceleration due to gravity, $\left[\mathrm{LT}^{-2}\right]$.

$f_{U f}$ probability density function of $u_{f}$.

$f_{Z g}$ probability density function of $z_{g}$.

$f_{\mathrm{T} f}$ probability density function of $\tau_{f}$.

$f_{\mathrm{T} g}$ probability density function of $\tau_{g}$.

$f_{\mathrm{T} g, Z g}$ joint probability density function of $\tau_{g}$ and $z_{g}$.

$h_{u}$ uniform flow depth, [L].

$t$ time, [T].

$u_{f}, v_{f}, w_{f}$ near-bed fluid velocity components, $\left[\mathrm{LT}^{-1}\right]$.

$u_{f}^{\prime}, v_{f}^{\prime}, w_{f}^{\prime}$ temporal fluctuations of near-bed fluid velocity components, $\left[\mathrm{LT}^{-1}\right]$.

$u_{\text {laser }}, v_{\text {laser }}, w_{\text {laser }}$ fluid velocity at the laser level, $\left[\mathrm{LT}^{-1}\right]$.

$u_{s}$ shear velocity, $\left[\mathrm{LT}^{-1}\right]$.

$z$ distance from the reference level, [L].

$z_{0}$ Zero average velocity level elevation for the log-law, [L].

$z_{\text {bed }}$ top of the bed grains elevation, [L].

$z_{f}$ fluid drag elevation, [L].

$z_{g}$ target particle elevation, [L].

$z_{\text {laser }}$ laser sheet elevation (= flow measurement elevation), [L].

$\Delta$ relative submerged density of sediments.

$\gamma$ specific weight of water, $\left[\mathrm{ML}^{-2} \mathrm{~T}^{-2}\right]$.

$\delta_{R}$ thickness of the roughness interface, [L].

$\mu_{u f}, \mu_{v f}, \mu_{w f}$ expected value of $u_{f}, v_{f}, w_{f},\left[\mathrm{LT}^{-1}\right]$.

$\mu_{u, \text { laser }}$ expected value of $u_{\text {laser }},\left[\mathrm{LT}^{-1}\right]$.

$\mu_{z g}$ expected value of $z_{g},[\mathrm{~L}]$.

$\mu_{\tau g}$ expected value of $\tau_{g},\left[\mathrm{ML}^{-1} \mathrm{~T}^{-2}\right]$.

$\nu$ kinematic viscosity, $\left[\mathrm{L}^{2} \mathrm{~T}^{-1}\right]$.

$\rho$ density of water, $\left[\mathrm{ML}^{-3}\right]$.

$\rho_{s}$ density of sediments, $\left[\mathrm{ML}^{-3}\right]$.

$\sigma_{g}$ geometrical standard deviation of the sediment distribution.

$\sigma_{u f}, \sigma_{v f}, \sigma_{w f}$ standard deviation of $u_{f}, v_{f}, w_{f},\left[\mathrm{LT}^{-1}\right]$.

$\sigma_{\text {u,laser }}$ standard deviation of $u_{\text {laser }},\left[\mathrm{LT}^{-1}\right]$.

$\sigma_{t m}$ geometrical standard deviation of travel time distribution.

$\sigma_{z g}$ standard deviation of $z_{g}$, [L].

$\sigma_{\tau g}$ standard deviation of $\tau_{g},\left[\mathrm{ML}^{-2} \mathrm{~T}^{-2}\right]$.

$\tau_{0}$ bed shear stress, $\left[\mathrm{ML}^{-2} \mathrm{~T}^{-2}\right]$.

$\tau_{f}$ fluid shear stress, $\left[\mathrm{ML}^{-2} \mathrm{~T}^{-2}\right]$.

$\tau_{g}$ critical shear stress, $\left[\mathrm{ML}^{-2} \mathrm{~T}^{-2}\right]$

$\chi_{\mu}$ scaling parameter for the mean velocity.

$\chi_{\sigma}$ scaling parameter for the standard deviation of velocity.

$\omega_{f}$ particle exposure to the fluid.

[42] Acknowledgments. Modeling and data analysis was carried out within the Project "PARTS: Probabilistic Assessment of the Retention and Transport of Sediments and Associated Pollutants in Rivers," funded by the EU Research Executive Agency via a IntraEuropean Fellowship to M. Tregnaghi under a Marie Curie action funding scheme. The writers would like to recognize the support of John Heald and Songdong Shao in the analysis of the DPM simulations.

\section{References}

Afzalimehr, H., and C. D. Rennie (2009), Determination of bed shear stress in gravel-bed rivers using boundary-layer parameters, Hydrol. Sci. $J ., 54(1), 147-159$, doi:10.1623/hysj.54.1.147.

Ancey, C., T. Böhm, M. Jodeau, and P. Frey (2006), Statistical description of sediment transport experiments, Phys. Rev. E, 74, 011302, doi:10.1103/ PhysRevE.74.011302.

Ancey, C., A. C. Davison, T. Böhm, M. Jodeau, and P. Frey (2008), Entrainment and motion of coarse particles in a shallow water stream down a steep slope, J. Fluid Mech., 595, 83-114, doi:10.1017/S0022112007008774.

Balachandar, S., and L. Sirovich (1991), Probability distribution functions in turbulent convection, Phys. Fluids A, 3(5), 919-927, doi:10.1063/ 1.857968 .

Bottacin-Busolin, A., S. J. Tait, A. Marion, A. Chegini, and M. Tregnaghi (2008), Probabilistic description of grain resistance from simultaneous flow field and grain motion measurements, Water Resour. Res., 44, W09419, doi:10.1029/2007WR006224.

Buffington, J. R., J. W. Kirchner, and W. E. Dietricht (1992), Friction angle measurements on a naturally formed gravel streambed: Implications for critical boundary shear stress, Water Resour. Res., 28(2), 411-425, doi:10.1029/91WR02529.

Cao, Z. (1997), Turbulent bursting-based sediment entrainment function, J. Hydraul. Eng., 123(3), 233-236, doi:10.1061/(ASCE)0733-9429 (1997)123:3(233)

Cheng, N. S., A. W. K. Law, and S. Y. Lim (2003), Probability distribution of bed particle instability, Adv. Water Resour., 26(4), 427-433, doi:10.1016/ S0309-1708(02)00184-7.

Church, M., M. A. Hassan, and J. F. Wolcott (1998), Stabilizing self-organized structures in gravel-bed stream channels: Field and experimental observations, Water Resour. Res., 34(11), 3169-3179, doi:10.1029/ 98WR00484

Cooper, J. R., and S. J. Tait (2010), Spatially representative velocity measurement over water-worked gravel beds, Water Resour. Res., 46, W11559, doi:10.1029/2009WR008465.

Dancey, C. L., P. Diplas, A. N. Papanicolaou, and M. Bala (2002), Probability of individual grain movement and threshold condition, J. Hydraul. Eng., 128(12), 1069-1075, doi:10.1061/(ASCE)0733-9429(2002)128:12(1069).

Dey, S. (1999), Sediment threshold, Appl. Math. Model., 23, 399-417, doi:10.1016/S0307-904X(98)10081-1.

Dinavahi, S. P. G., K. S. Breuer, and M. Sirovich (1995), Universality of probability density functions in turbulent channel flow, Phys. Fluids, 7(5), 1122-1129, doi:10.1063/1.868553.

Diplas, P., C. L. Dancey, A. O. Celik, M. Valyrakis, K. Greer, and T. Akar (2008), The role of impulse on the initiation of particle movement under turbulent flow conditions, Science, 322(5902), 717-720, doi:10.1126/ science. 1158954

Dittrich, A., and K. Koll (1997), Velocity field and resistance of flow over rough surface with large and small relative submergence, Int. J. Sediment Res., 12(3), 21-33.

Drake, T. G., R. L. Shreve, W. E. Dietrich, P. J. Whiting, and L. Leopold (1988), Bedload transport of fine gravel observed by motion picture, J. Fluid Mech., 192, 193-217, doi:10.1017/S0022112088001831.

Einstein, H. A. (1942), Formulas for the transportation of bed load, Trans. Am. Soc. Civ. Eng., 107, 561-597.

Fenton, J. D., and J. E. Abbott (1977), Initial movement of grains on a stream bed: The effect of relative protrusion, Proc. R. Soc. London, Ser. A, 352, 523-537, doi:10.1098/rspa.1977.0014.

Heald, J. G. C. I. K. McEwan, and S. J. Tait (2004), Sediment transport over a flat bed in a unidirectional flow: Simulations and validation, Philos. Trans. R. Soc. London A, 362, 1973-1986, doi:10.1098/rsta.2004.1426.

Jiménez, J. (1998), Turbulent velocity fluctuations need not be Gaussian, J. Fluid Mech., 376, 139-147, doi:10.1017/S0022112098002432.

Johnston, C. E., E. D. Andrews, and J. Pitlick (1998), In situ determination of particle friction angles of fluvial gravels, Water Resour. Res., 34(8), 2017-2030, doi:10.1029/98WR00312.

Kirchner, J. W., W. E. Dietricht, F. Iseya, and H. Ikeda (1990), The variability of critical shear stress, friction angle, and grain protrusion in water-worked sediments, Sedimentology, 37, 647-672, doi:10.1111/j.1365-3091.1990. tb00627.x.

Kironoto, B. A., and W. H. Graf (1994), Turbulence characteristics in rough uniform open-channel flow, Proc. Ice Water Mar. Energy, 106, 333-344.

Lajeunesse, E., L. Malverti, and F. Charru (2010), Bed load transport in turbulent flow at the grain scale: Experiments and modeling, J. Geophys. Res., 115, F04001, doi:10.1029/2009JF001628.

Leeder, M. R. (1983), On the dynamics of sediment suspension by residual Reynolds stresses-confirmation of Bagnold's theory, Sedimentology, 30, 485-491, doi:10.1111/j.1365-3091.1983.tb00687.x. 
Marion, A., S. J. Tait, and I. K. McEwan (2003), Analysis of small-scale gravel bed topography during armouring, Water Resour. Res., 39(12), 1334, doi:10.1029/2003WR002367.

McEwan, I. K., and J. G. C. Heald (2001), Discrete particle modeling of entrainment from flat uniformly sized sediment beds, J. Hydraul. Eng., 127(7), 588-597, doi:10.1061/(ASCE)0733-9429(2001)127:7(588).

Nelson, J. M., R. L. Shreve, S. R. McLean, and T. G. Drake (1995), Role of near bed turbulence structure in bed load transport and bed mechanics, Water Resour. Res., 31(8), 2071-2086, doi:10.1029/95WR00976.

Nezu, I., and H. Nakagawa (1993), Turbulence in Open-Channel Flows, Balkema, Rotterdam, Netherlands.

Nikora, V., D. K. Goring, I. K. McEwan, and G. Griffith (2001), Spatially averaged open-channel flow over rough bed, J. Hydraul. Eng., 127(2), 123-133, doi:10.1061/(ASCE)0733-9429(2001)127:2(123).

Nikora, V., K. Koll, I. K. McEwan, S. McLean, and A. Dittrich (2004), Velocity distribution in the roughness layer of rough-bed flows, $J$. Hydraul. Eng., 130(10), 1036-1042, doi:10.1061/(ASCE)0733-9429 (2004)130:10(1036).

Papanicolaou, A., P. Diplas, C. Dancey, and M. Balakrishnan (2001), Surface roughness effects in near-bed turbulence: Implications to sediment transport, J. Eng. Mech., 127(3), 211-218, doi:10.1061/(ASCE)07339399(2001)127:3(211).

Papanicolaou, A., P. Diplas, N. Evaggelopoulos, and S. Fotopoulos (2002), Stochastic incipient motion criterion for spheres under various bed packing conditions, J. Hydraul. Eng., 128(4), 369-380, doi:10.1061/(ASCE) 0733-9429(2002)128:4(369).

Radice, A., F. Ballio, and V. Nikora (2009), On statistical properties of bed load sediment concentration, Water Resour. Res., 45, W06501, doi:10.1029/ 2008WR007192.

Rennie, C. D., and P. V. Villard (2004), Site specificity of bed load measurement using an acoustic Doppler current profiler, J. Geophys. Res., 109, F03003, doi:10.1029/2003JF000106.
Schmeeckle, M. W., and J. M. Nelson (2003), Direct numerical simulation of bedload transport using a local, dynamic boundary condition, Sedimentology, 50, 279-301, doi:10.1046/j.1365-3091.2003.00555.x.

Schmeeckle, M. W., J. M. Nelson, and R. L. Shreve (2007), Forces on stationary particles in near-bed turbulent flows, J. Geophys. Res., 112, F02003, doi:10.1029/2006JF000536.

Shvidchenko, A. B., and G. Pender (2000), Flume study of the effect of relative depth on the incipient motion of coarse uniform sediments, Water Resour. Res., 36(2), 619-628, doi:10.1029/1999WR900312.

Townsend, A. A. (1976), The Structure of Turbulent Shear Flows, Cambridge Univ. Press, Cambridge, U. K.

Tregnaghi, M., A. Busolin-Bottacin, S. J. Tait, and A. Marion (2010), 3D nearbed flow field measurements at low sediment transport rates, paper presented at River Flow 2010, International Conference on Fluvial Hydraulics, Int. Assoc. of Hydraul. Eng. and Res., Braunschweig, Germany, 8-10 Sept.

Tregnaghi, M., A. Bottacin-Busolin, A. Marion, and S. Tait (2012), Stochastic determination of entrainment risk in uniformly sized sediment beds at low transport stages: 1. Theory, J. Geophys. Res., 117, F04004, doi:10.1029/2011JF002134

Valyrakis, M., P. Diplas, C. L. Dancey, K. Greer, and A. O. Celik (2010), The role of instantaneous force magnitude and duration on particle entrainment, J. Geophys. Res., 115, F02006, doi:10.1029/2008JF001247.

Valyrakis, M., P. Diplas, and C. L. Dancey (2011), Entrainment of coarse grains in turbulent flows: An extreme value theory approach, Water Resour. Res., 47, W09512, doi:10.1029/2010WR010236.

Wiberg, J. R., and D. R. Smith (1987), Calculations of the critical shear stress for motion of uniform and heterogeneous sediments, Water Resour. Res., 23(8), 1471-1480, doi:10.1029/WR023i008p01471.

Wu, F. C., and M. R. Jiang (2007), Numerical investigation of the role of turbulent bursting in sediment entrainment, J. Hydraul. Eng., 133(3), 329-334, doi:10.1061/(ASCE)0733-9429(2007)133:3(329). 